\title{
Genome-Wide Identification and Expression Profile of TPS Gene Family in Dendrobium officinale and the Role of DoTPS10 in Linalool Biosynthesis
}

\author{
Zhenming Yu ${ }^{1,2,+}$, Conghui Zhao ${ }^{1,3,+}$, Guihua Zhang ${ }^{1}{ }^{1}$, Jaime A. Teixeira da Silva ${ }^{4}$ and \\ Jun Duan 1,2,* \\ 1 Guangdong Provincial Key Laboratory of Applied Botany \& Key Laboratory of South China Agricultural \\ Plant Molecular Analysis and Genetic Improvement, South China Botanical Garden, Chinese Academy of \\ Sciences, Guangzhou 510650, China; zhenming311@scbg.ac.cn (Z.Y.); zhaoconghui@scbg.ac.cn (C.Z.); \\ zhanggh@scbg.ac.cn (G.Z.) \\ 2 Center of Economic Botany, Core Botanical Gardens, Chinese Academy of Sciences, \\ Guangzhou 510650, China \\ 3 University of Chinese Academy of Sciences, No. 19A Yuquan Road, Beijing 100049, China \\ 4 Independent Researcher, P.O. Box 7, Miki-cho Post Office, Ikenobe 3011-2, Kagawa-ken 761-0799, Japan; \\ jaimetex@yahoo.com \\ * Correspondence: duanj@scib.ac.cn; Tel.: +86-020-37252978 \\ + These authors contributed equally to this work.
}

Received: 25 June 2020; Accepted: 28 July 2020; Published: 30 July 2020

\begin{abstract}
Terpene synthase (TPS) is a critical enzyme responsible for the biosynthesis of terpenes, which possess diverse roles in plant growth and development. Although many terpenes have been reported in orchids, limited information is available regarding the genome-wide identification and characterization of the TPS family in the orchid, Dendrobium officinale. By integrating the D. officinale genome and transcriptional data, 34 TPS genes were found in D. officinale. These were divided into four subfamilies (TPS-a, TPS-b, TPS-c, and TPS-e/f). Distinct tempospatial expression profiles of DoTPS genes were observed in 10 organs of D. officinale. Most DoTPS genes were predominantly expressed in flowers, followed by roots and stems. Expression of the majority of DoTPS genes was enhanced following exposure to cold and osmotic stresses. Recombinant DoTPS10 protein, located in chloroplasts, uniquely converted geranyl diphosphate to linalool in vitro. The DoTPS10 gene, which resulted in linalool formation, was highly expressed during all flower developmental stages. Methyl jasmonate significantly up-regulated DoTPS10 expression and linalool accumulation. These results simultaneously provide valuable insight into understanding the roles of the TPS family and lay a basis for further studies on the regulation of terpenoid biosynthesis by DoTPS in D. officinale.
\end{abstract}

Keywords: terpene synthase; terpenes; methyl jasmonate; abiotic stress; orchids

\section{Introduction}

Terpenes, which are derived biosynthetically from two isomeric 5-carbon building blocks, dimethylallyl diphosphate (DMAPP) and isopentenyl diphosphate (IPP), are the largest family of plant secondary metabolites [1]. Plant terpenes play vital roles in attracting insect pollinators [2], plant defense response [1,3], plant-plant interactions [4], and the mediation of interactions with various ecological habitats [5]. The high volatility of terpene compounds promotes the scent in orchids. For instance, geraniol and linalool are major floral scent compounds in Phalaenopsis bellina [6,7]. Orchid floral volatiles, as well as flower color, shape, and fragrance are key horticultural ornamental traits in orchids, and also serve to attract pollinators in various ecological habitats [6]. 
The biosynthetic pathway of volatile terpenes is well characterized in plants (Figure 1). Generally, the C5 precursors DMAPP and IPP are formed, and the direct precursors farnesyl diphosphate (FPP), geranyl diphosphate (GPP), and geranylgeranyl diphosphate (GGPP) are generated. Subsequently, plant terpenes are biosynthesized by terpene synthase (TPS), which converts FPP to sesquiterpene in the cytosol via the mevalonic acid (MVA) pathway, and GPP and GGPP to monoterpenes and diterpenes, respectively in plastids by the methylerythritol phosphate (MEP) pathway $[1,8]$. TPS is positioned at the branch point of the isoprenoid pathway, and is a key enzyme for terpenoid synthesis.

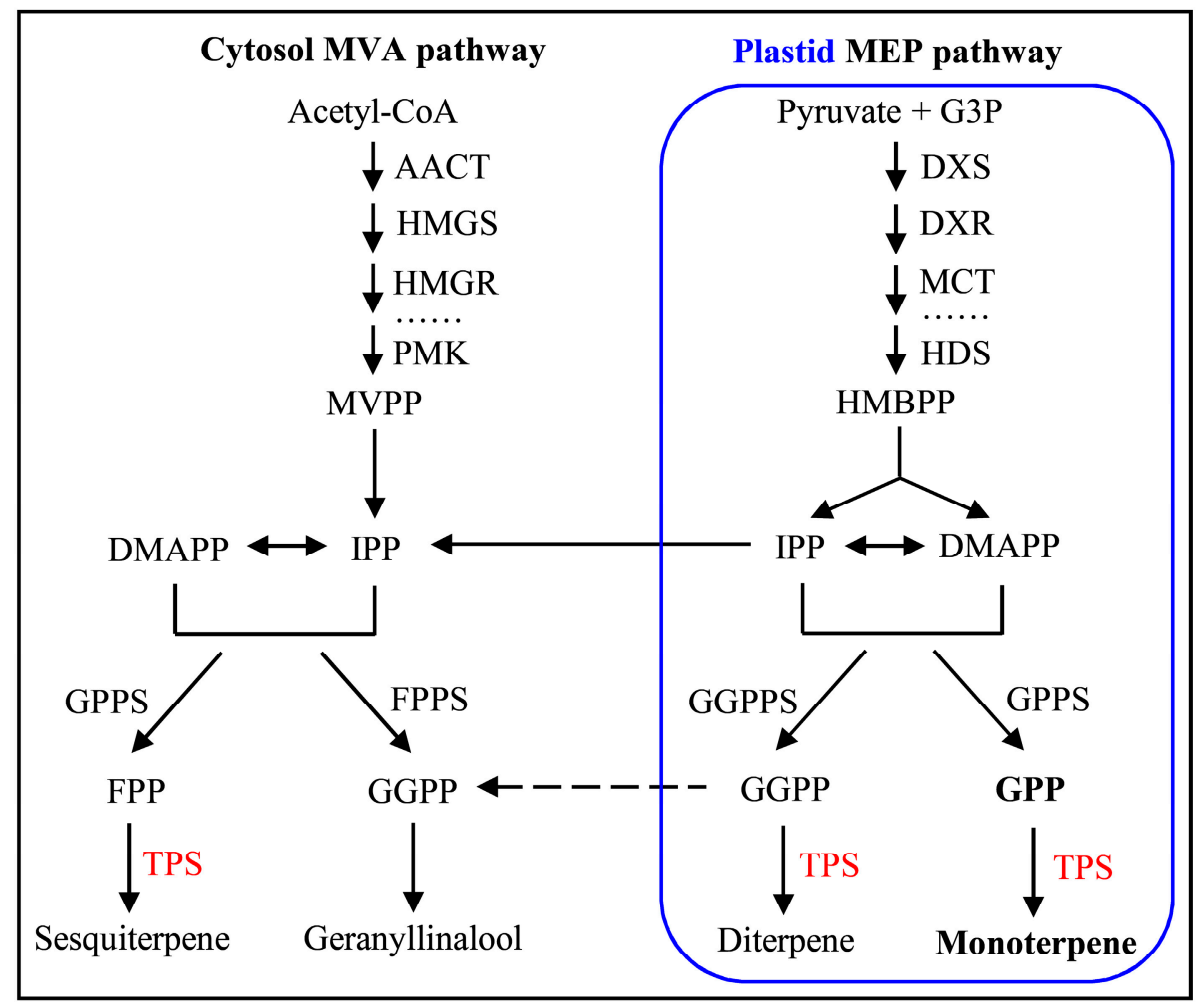

Figure 1. The pathway of terpene synthase genes responsible for the formation of terpenes in planta [3]. Terpenes are biosynthesized by the cytosol mevalonic acid (MVA) and the plastid methylerythritol phosphate (MEP) pathways, the former giving rise to sesquiterpenes and geranyllinalool, and the latter to monoterpenes and diterpenes. AACT, acetyl-CoA acetyltransferase; DMAPP, dimethylallyl pyrophosphate; DXS, 1-deoxy-d-xylulose 5-phosphate synthase; DXR, 1-deoxy-d-xylulose 5-phosphate reductoisomerase; FPP, farnesyl pyrophosphate; FPPS, FPP synthase; G3P, glyceraldehyde 3-phosphate; GGPP, geranylgeranyl pyrophosphate; GGPPS, GGPP synthase; GPP, geranyl pyrophosphate; GPPS, GPP synthase; HDS, 4-hydroxy-3-methylbut-2-en-1-yl diphosphate synthase; HMBPP, (E)-4-hydroxy-3-methylbut-2-en-1-yl diphosphate; HMGR, hydroxymethylglutaryl-CoA reductase; HMGS, hydroxymethylglutaryl-CoA synthase; IPP, isopentenyl pyrophosphate; MCT, 2-C-methyl-d-erythritol 4-phosphate cytidylyltransferase; MVPP, mevalonate 5-pyrophosphate; PMK, phosphomevalonate kinase; TPS, terpene synthase.

Each TPS is characterized by two conserved domains, PF03936 (C-terminal) and PF01397 (N-terminal) [9], as indicated in the Pfam (http://pfam.xfam.org/) database. The TPS family is phylogenetically classified into seven subfamilies (TPS-a, TPS-b, TPS-c, TPS-d, TPS-e/f, TPS-g, and TPS-h) [1]. Among them, TPS-a encodes sesquiterpene synthase that is found in both dicotyledonous and monocotyledonous plants. The angiosperm-specific TPS-b encodes monoterpene synthase with a $\mathrm{R}(\mathrm{R}) \mathrm{X}_{8} \mathrm{~W}$ motif that catalyzes the isomerization cyclization reaction. TPS-c is deemed to belong to the ancestral clade and catalyzes copalyl diphospate synthase. The gymnosperm-specific TPS- $d$ performs several functions, as diterpene, monoterpene, and sesquiterpene synthases. TPS-e/f encodes copalyl diphosphate/kaurene synthases, which are critical enzymes for the production of 
gibberellic acid. Another angiosperm-specific TPS-g encodes monoterpene synthase without the $\mathrm{R}(\mathrm{R}) \mathrm{X}_{8} \mathrm{~W}$ motif. TPS-h is only observed in Selaginella moellendorffii [1,9-11]. In addition, TPS harbors conserved structural features such as DDxxD, NSE/DTE, and R(R) $\mathrm{X}_{8} \mathrm{~W}$ motifs [1].

To date, TPS gene families have been identified at the genome-wide level in various plant species, including Arabidopsis thaliana [12], Camellia sinensis [13], Daucus carota [14], Eucalyptus globulus and E. grandis [15], Malus domestica [16], Solanum lycopersicum [17], Selaginella moellendorffii [18], and Vitis vinifera [19]. Orchids form one of the largest families of flowering plants, and their metabolic profile contains various terpenes [7]. Only a few TPS genes have been identified thus far in orchids. PbTPS5 and PbTPS10 might be involved in monoterpene biosynthesis in Phalaenopsis bellina [20]. FhTPS1 catalyzes the formation of linalool, while FhTPS4, FhTPS6, and FhTPS7 are bifunctional enzymes that can simultaneously recognize FPP and GPP as substrates [21]. However, no comprehensive study about TPS genes in Dendrobium officinale exists.

D. officinale is an endangered orchid native to South and Southeast Asia, and is used for medicinal purposes in Chinese culture [22]. Moreover, D. officinale is a unique orchid because it grows on rocks, trees, or even cliffs. In order to adapt to harsh growth conditions, terpene compounds are synthesized [6,22-25]. Therefore, it is necessary to characterize the TPS gene family and study the roles of TPS in D. officinale. These findings will provide a valuable reference about the terpene biosynthetic pathway in orchids.

\section{Results}

\subsection{Genome-Wide Identification and Features of TPS Proteins in D. officinale}

To systematically identify the TPS genes in D. officinale, a hidden Markov model (HMM) profile of the conserved C-terminal (PF03936) and N-terminal (PF01397) domains in the TPS protein was used as a BLAST query against the D. officinale genome database [23]. After the removal of redundant sequences, 34 TPS genes were obtained (Table 1). The open reading frame (ORF) of DoTPS ranged from 378 (DoTPS12) to $2571 \mathrm{bp}$ (DoTPS4), the deduced length of the amino acids ranged from 125 (DoTPS12) to 856 aa (DoTPS4), and molecular weight (Mw) ranged from 14.98 (DoTPS12) to $100.05 \mathrm{kDa}$ (DoTPS4). The theoretical isoelectric point (pI) values of DoTPS proteins ranged from 4.94 (DoTPS19) to 7.18 (DoTPS4). The calculated grand average of hydrophobicity (GRAVY) values, ranging from -0.429 (DoTPS4) to 0.013 (DoTPS19), indicated most DoTPS proteins were hydrophilic, except for DoTPS19 with a GRAVY value $>0$. In addition, the aliphatic index (AI) of DoTPS proteins ranged from 80.35 (DoTPS4) to 110.46 (DoTPS4), and the instability index (II) of these proteins ranged from 33.82 (DoTPS20) to 51.56 (DoTPS31). According to three widely used predictors (AtSubP [26], Plant-mPLoc [27], and pLoc-mPlant [28], all having good accuracy with greater than 70\%), 14/34 DoTPS proteins were targeted to the chloroplast, other 20 DoTPS proteins were targeted to chloroplast or cytoplasm (Table 1, Tables S1-S3), suggesting that different predictors produce different results, and it was better to verify by experimental results. The prediction of secondary structures demonstrated that $\alpha$-helixes and random coils were dominant in all DoTPS proteins, followed by extended strands and $\beta$-turns, accounting for on average $68.68,23.82,4.33$, and 3.17\%, respectively (Table S4).

Table 1. Information of the plant TPS gene family in D. officinale.

\begin{tabular}{|c|c|c|c|c|c|c|c|c|c|}
\hline Name & Gene ID ${ }^{1}$ & $\begin{array}{c}\mathrm{ORF}^{2} \\
(\mathrm{bp})\end{array}$ & $\begin{array}{c}\mathbf{A A}^{3} \\
\text { (aa) }\end{array}$ & $\mathrm{pI}^{4}$ & $\begin{array}{l}\mathrm{Mw}^{5} \\
(\mathrm{kDa})\end{array}$ & $\mathrm{AI}^{6}$ & II $^{7}$ & GRAVY $^{8}$ & Localization $^{9}$ \\
\hline DoTPS1 & Dca014928 & 960 & 319 & 6.31 & 36.82 & 90.78 & 48.81 & -0.342 & Chloroplast ${ }^{\mathrm{a}, \mathrm{b}, \mathrm{c}}$ \\
\hline DoTPS2 & Dca000724 & 1902 & 633 & 6.47 & 74.02 & 88.44 & 43.11 & -0.260 & Chloroplast ${ }^{\mathrm{a}, \mathrm{b}, \mathrm{c}}$ \\
\hline DoTPS3 & Dca000725 & 1827 & 608 & 5.46 & 70.52 & 89.67 & 41.24 & -0.231 & Chloroplast ${ }^{\mathrm{a}, \mathrm{b}, \mathrm{c}}$ \\
\hline DoTPS4 & Dca022838 & 2571 & 856 & 7.18 & 100.05 & 80.35 & 47.78 & -0.429 & Chloroplast ${ }^{\mathrm{a}, \mathrm{b}, \mathrm{c}}$ \\
\hline DoTPS5 & Dca003141 & 1692 & 563 & 5.11 & 65.84 & 98.86 & 42.13 & -0.228 & Chloroplast ${ }^{\mathrm{a}, \mathrm{b}} /$ Cytoplasm $^{\mathrm{c}}$ \\
\hline DoTPS6 & Dca019411 & 1521 & 506 & 5.13 & 59.48 & 93.68 & 43.71 & -0.133 & Chloroplast ${ }^{\mathrm{a}, \mathrm{b}} /$ Cytoplasm $\mathrm{c}$ \\
\hline DoTPS7 & Dca003139 & 1692 & 563 & 5.67 & 65.72 & 92.49 & 39.06 & -0.266 & Chloroplast $\mathrm{a}, \mathrm{b} /$ Cytoplasm $\mathrm{c}$ \\
\hline DoTPS8 & Dca028160 & 579 & 192 & 6.83 & 22.63 & 100.62 & 45.31 & -0.121 & $\begin{array}{c}\text { Chloroplast a/Unknown } \\
\text { b/Cytoplasm }{ }^{c}\end{array}$ \\
\hline DoTPS9 & Dca019412 & 1665 & 554 & 5.03 & 64.93 & 92.94 & 39.76 & -0.189 & Chloroplast $^{\mathrm{a}, \mathrm{b}} /$ Cytoplasm $^{\mathrm{c}}$ \\
\hline
\end{tabular}


Table 1. Cont.

\begin{tabular}{|c|c|c|c|c|c|c|c|c|c|}
\hline Name & Gene ID $^{1}$ & $\begin{array}{c}\text { ORF }^{2} \\
(\mathrm{bp})\end{array}$ & $\begin{array}{c}\mathbf{A A}^{3} \\
\text { (aa) }\end{array}$ & $\mathrm{pI}^{4}$ & $\begin{array}{l}\mathrm{Mw}^{5} \\
(\mathrm{kDa})\end{array}$ & $\mathrm{AI}^{6}$ & II $^{7}$ & GRAVY $^{8}$ & Localization $^{9}$ \\
\hline DoTPS10 & Dca007746 & 1797 & 598 & 5.73 & 69.73 & 93.61 & 47.41 & -0.242 & Chloroplast $^{\mathrm{a}, \mathrm{b}} /$ Cytoplasm $^{\mathrm{c}}$ \\
\hline DoTPS11 & Dca022749 & 696 & 231 & 5.13 & 27.40 & 104.20 & 49.98 & -0.045 & Chloroplast $^{\mathrm{a}, \mathrm{b}} /$ Cytoplasm $^{\mathrm{c}}$ \\
\hline DoTPS12 & Dca024936 & 378 & 125 & 5.64 & 14.98 & 98.32 & 40.18 & -0.326 & Chloroplast ${ }^{\mathrm{a}} /$ Cytoplasm $\mathrm{b}, \mathrm{c}$ \\
\hline DoTPS13 & Dca026570 & 1659 & 552 & 5.59 & 64.94 & 96.97 & 38.16 & -0.266 & Chloroplast $\mathrm{a}, \mathrm{b}, \mathrm{c}$ \\
\hline DoTPS14 & Dca005188 & 2550 & 849 & 6.71 & 98.69 & 86.21 & 46.66 & -0.352 & Chloroplast ${ }^{\mathrm{a}, \mathrm{b}, \mathrm{c}}$ \\
\hline DoTPS15 & Dca025698 & 1659 & 552 & 5.31 & 64.82 & 89.60 & 44.73 & -0.284 & Chloroplast ${ }^{\mathrm{a}, \mathrm{b}, \mathrm{c}}$ \\
\hline DoTPS16 & Dca016979 & 1650 & 549 & 5.62 & 64.23 & 91.62 & 36.94 & -0.374 & Chloroplast $^{\mathrm{a}, \mathrm{b}} /$ Cytoplasm $^{\mathrm{c}}$ \\
\hline DoTPS17 & Dca008309 & 1653 & 550 & 5.42 & 64.89 & 97.13 & 47.01 & -0.233 & Chloroplast ${ }^{\mathrm{a}, \mathrm{b}, \mathrm{c}}$ \\
\hline DoTPS18 & Dca011215 & 1674 & 557 & 5.36 & 64.80 & 95.10 & 44.24 & -0.302 & Chloroplast ${ }^{\mathrm{a}, \mathrm{b}, \mathrm{c}}$ \\
\hline DoTPS19 & Dca010855 & 1446 & 481 & 4.94 & 55.95 & 110.46 & 34.63 & 0.013 & Chloroplast ${ }^{\mathrm{a}, \mathrm{b}, \mathrm{c}}$ \\
\hline DoTPS20 & Dca026890 & 1749 & 582 & 5.20 & 68.07 & 92.84 & 33.82 & -0.295 & Chloroplast ${ }^{\mathrm{a}, \mathrm{b}, \mathrm{c}}$ \\
\hline DoTPS21 & Dca007747 & 1797 & 598 & 5.62 & 69.60 & 94.92 & 48.15 & -0.224 & Chloroplast $^{\mathrm{a}, \mathrm{b}} /$ Cytoplasm $^{\mathrm{c}}$ \\
\hline DoTPS22 & Dca003142 & 1692 & 563 & 5.24 & 65.61 & 95.26 & 43.73 & -0.245 & Chloroplast $^{\mathrm{a}, \mathrm{b}} /$ Cytoplasm $^{\mathrm{c}}$ \\
\hline DoTPS23 & Dca011214 & 1674 & 557 & 5.22 & 65.03 & 90.36 & 37.30 & -0.331 & Chloroplast $^{\mathrm{a}, \mathrm{b}} /$ Cytoplasm $^{\mathrm{c}}$ \\
\hline DoTPS24 & Dca000728 & 1386 & 461 & 6.38 & 53.78 & 93.08 & 38.16 & -0.180 & Chloroplast $^{\mathrm{a}, \mathrm{b}} /$ Cytoplasm $^{\mathrm{c}}$ \\
\hline DoTPS25 & Dca013782 & 1794 & 597 & 5.31 & 69.67 & 94.61 & 44.76 & -0.274 & Chloroplast ${ }^{\mathrm{a}, \mathrm{b}} /$ Cytoplasm $^{\mathrm{c}}$ \\
\hline DoTPS26 & Dca026369 & 1650 & 549 & 5.42 & 64.61 & 90.73 & 42.39 & -0.438 & Chloroplast $^{\mathrm{a}, \mathrm{b}} /$ Cytoplasm $^{\mathrm{c}}$ \\
\hline DoTPS27 & Dca000723 & 1938 & 645 & 5.89 & 74.89 & 91.74 & 48.68 & -0.240 & Chloroplast $^{\mathrm{a}, \mathrm{b}} /$ Cytoplasm $^{\mathrm{c}}$ \\
\hline DoTPS28 & Dca003295 & 1863 & 620 & 5.91 & 72.57 & 92.35 & 47.89 & -0.262 & Chloroplast $^{\mathrm{a}, \mathrm{b}} /$ Cytoplasm $^{\mathrm{c}}$ \\
\hline DoTPS29 & Dca018407 & 1653 & 550 & 5.57 & 64.68 & 95.89 & 50.10 & -0.251 & Chloroplast $^{\mathrm{a}, \mathrm{b}} /$ Cytoplasm $^{\mathrm{c}}$ \\
\hline DoTPS30 & Dca013784 & 1377 & 458 & 5.07 & 53.35 & 93.52 & 38.64 & -0.254 & Chloroplast $\mathrm{a}, \mathrm{b}$ \\
\hline DoTPS31 & Dca016966 & 1089 & 362 & 7.07 & 41.65 & 99.70 & 51.56 & -0.193 & Chloroplast ${ }^{\mathrm{a}, \mathrm{b}, \mathrm{c}}$ \\
\hline DoTPS32 & Dca018946 & 2433 & 810 & 5.75 & 91.13 & 88.99 & 46.05 & -0.169 & Chloroplast ${ }^{\mathrm{a}, \mathrm{b}, \mathrm{c}}$ \\
\hline DoTPS33 & Dca017971 & 1536 & 511 & 5.57 & 58.70 & 97.18 & 42.66 & -0.109 & Chloroplast $^{\mathrm{a}, \mathrm{b}} /$ Cytoplasm $^{\mathrm{c}}$ \\
\hline DoTPS34 & Dca020940 & 1797 & 598 & 5.19 & 69.59 & 91.52 & 45.72 & -0.280 & Chloroplast $\mathrm{a}, \mathrm{b}, \mathrm{c}$ \\
\hline
\end{tabular}

${ }^{1}$ Gene ID, it is annotated in D. officinale genome [23]; ${ }^{2} \mathrm{ORF}$, open reading frame; ${ }^{3} \mathrm{AA}$, amino acid; ${ }^{4} \mathrm{pI}$, theoretical isoelectric point; ${ }^{5} \mathrm{Mw}$, molecular weight; ${ }^{6} \mathrm{AI}$, aliphatic index; ${ }^{7} \mathrm{II}$, instability index; ${ }^{8} \mathrm{GRAVY}$, grand average of hydrophobicity; ${ }^{9}$ Localization is predicted by Plant-mPLoc [27] (Table S1, http://www.csbio.sjtu.edu.cn/bioinf/plantmulti/), AtSubP [26] (Table S2, http://bioinfo3.noble.org/AtSubP/), and pLoc-mPlant [28] (Table S3, http://www.jcibioinfo.cn/pLoc-mPlant/) tools. ${ }^{a}, b, c$ indicates the result of Plant-mPLoc, AtSubP, and pLoc-mPlant, respectively.

\subsection{Analysis of Conserved Motifs and Gene Structure}

Since analysis of gene structure will facilitate an understanding of gene evolution and possible roles, the structure of DoTPS genes in D. officinale was investigated (Figure 2A). The amount of exons ranged from 2 to 14, with an average of 6.6 for all DoTPS genes. DoTPS32 contained the most exons (14), whereas DoTPS8 and DoTPS12 harbored the fewest exons (2). The majority of DoTPS genes (52.9\%) had seven exons. Apart from DoTPS7, -20,-24,-27, and -28, most of the genes that clustered in the same group generally possessed a similar exon-intron structure, especially in terms of intron number and exon length (Figure 2A). This conserved exon-intron structure within each cluster was in agreement with the classification of DoTPS genes in a neighbor-joining (NJ) phylogenetic tree based on DoTPS sequences.

To further elucidate the structural and functional features of DoTPS, 20 conserved motifs of the DoTPS proteins were identified using MEME software (Figure 2B). The lengths of these motifs ranged from 15 to 47 amino acids (Figure S1; Table S5). DoTPS3 contained the most motifs (18/20) while DoTPS31 had only two motifs. Motif 6 was found in all DoTPS proteins, except DoTPS12. Motifs 5 and 10 were the second most common DoTPS proteins (32/34), followed by motifs 1 and $2(31 / 34)$. DoTPS1, -8 , and -31 did not contain the DDxxD motif (motif 1), and DoTPS1, -12, and -31 did not contain the $\mathrm{R}(\mathrm{R}) \mathrm{X}_{8} \mathrm{~W}$ (motif 2 ) motif. Intriguingly, motif 14 was found in the cluster containing DoTPS5, $-6,-7,-9,13,-15,-16,-17,-22,-26$, and -29 . Motifs 17 and 20 were particularly abundant in the group containing DoTPS2, $-3,-10,-18,-19,-20,-21,-23,-25,-27,-28,-30,-32$, and -34 . Motif 20 only existed in the cluster that included DoTPS4, -14 , and -32. Motif 18 was only observed in a small branch that harbored DoTPS2, -3, -24, and -27 (Figure 2B). Despite the different types of motifs among clusters, DoTPS proteins within the same cluster generally possessed similar motifs. The diversity of DoTPS phylogenetic grouping patterns was likely influenced by the gene structure and the location of motifs. 


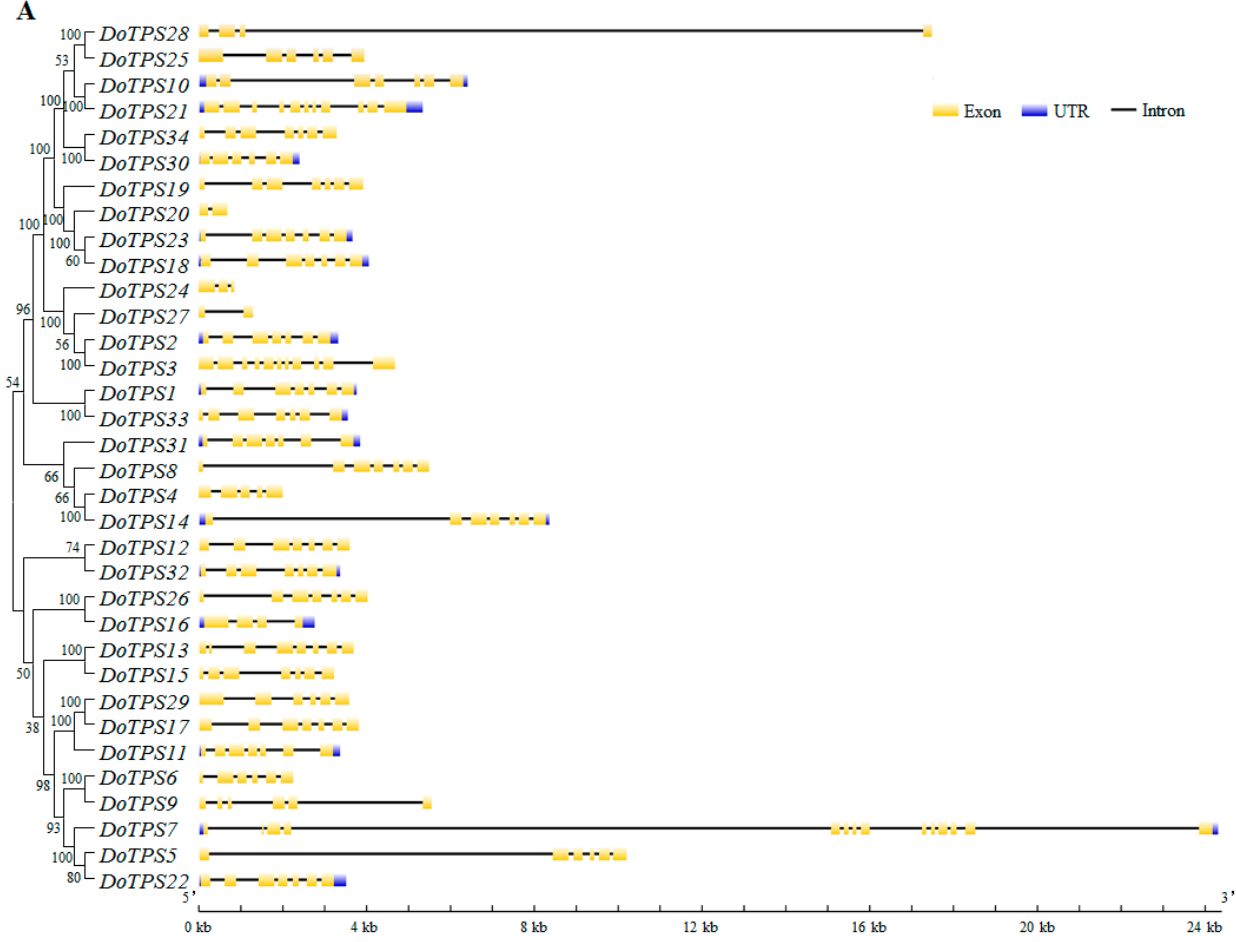

A

B

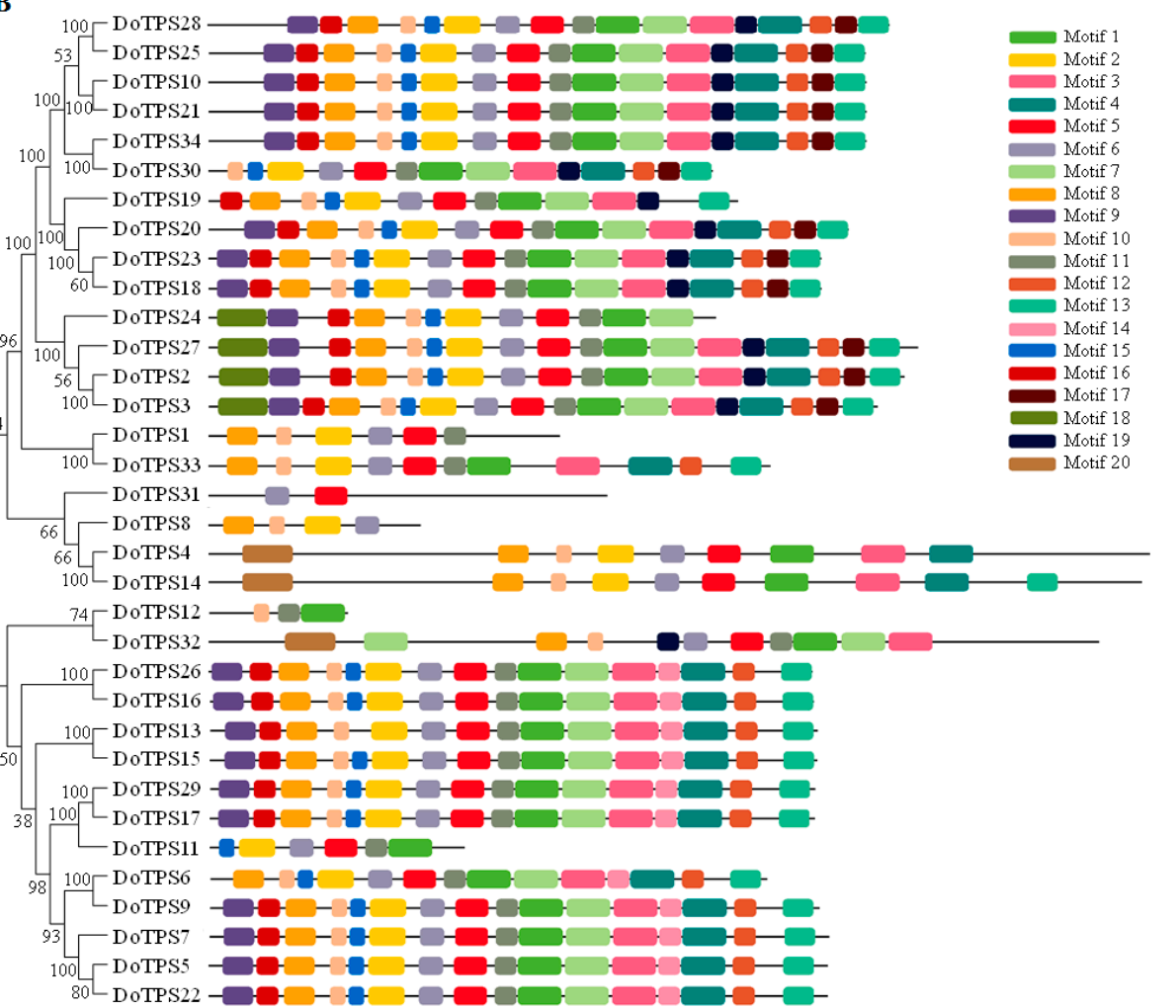

Figure 2. Phylogenetic relationships, exon-intron structure, and protein domain analysis of DoTPS genes in D. officinale. (A) Phylogenetic relationships and exon-intron structure of DoTPS genes. Exon-intron distribution was performed using GSDS 2.0 server (http://gsds.cbi.pku.edu.cn/). Yellow boxes indicate exons, black lines indicate introns. Blue boxes represent upstream/downstream-untranslated regions. (B) Phylogenetic relationships and motif structures of DoTPS genes. Phylogenetic tree was generated with MEGA 7.0 using the NJ method. Twenty classical motifs in DoTPS proteins were analyzed by the MEME tool. The width of each motif ranged from 15 to 47 amino acids. Different color blocks represented different motifs. 


\subsection{Phylogenetic Analysis of DoTPS Genes in D. officinale}

To gain further insight into the evolutionary relationships among the TPS subfamilies, an unrooted phylogenetic tree was constructed using the neighbor-joining (NJ) method implemented in MEGA 7.0 with the Jones-Taylor-Thornton (JTT) model based on multiple sequence alignment of TPS members from Abies grandis, A. thaliana, Apostasia shenzhenica, D. officinale, Oryza sativa, Phalaenopsis equestris, Populus trichocarpa, Selaginella moellendorffii, Solanum lycopersicum, and Sorghum bicolor (Figure 3, Table S6). The phylogenetic tree demonstrates that TPS proteins were clustered into seven subfamilies (TPS-a, TPS-b, TPS-c, TPS-d, TPS-e/f, TPS-g, and TPS-h) according to the published report $[1,15]$. Thirty-four DoTPS proteins appeared in only four groups (TPS-a, TPS-b, TPS-c, and TPS-e/f, 14, 16, 1 , and 3, respectively), and $88.2 \%$ of them belonged to TPS-a or TPS-b subgroups. Similarly, there were 20 and 9 TPS proteins present in P. equestris and A. shenzhenica, the amount of TPS-a, and TPS-b accounted for $12 / 20$, and $7 / 9$, respectively (Figure 3). This phenomenon was similar to that in A. thaliana and O. sativa [1,10]. Remarkably, in the TPS-a group, dicotyledonous and monocotyledonous plants formed distinct subgroups, which were observed previously [1,15] and termed them as TPS-a1 (dicots) and TPS-a2 (monocots), suggesting that the TPS-a genes evolved independently. Similar to the TPS-a group, the TPS-c group was further divided into dicot and monocot subclades. Consistent with a previous report, the TPS-d subfamily was specific to gymnosperms, and the TPS-h subfamily was only observed in S. moellendorffii $[1,11,12,17]$, inferring that they might play a particular role in these species.

Multiple sequence alignment of DoTPS proteins was further analyzed. As illustrated in Figure 4, the arginine-tryptophan motif, $\mathrm{R}(\mathrm{R}) \mathrm{X}_{8} \mathrm{~W}$, was found in all the DoTPS-a and DoTPS-b proteins, except DoTPS8, at the $N$-terminus. It plays a role in initiation of the isomerization cyclization reaction $[1,10]$. However, the arginine-tryptophan motif, $\mathrm{R}(\mathrm{R}) \mathrm{X}_{8} \mathrm{~W}$, varied or was even absent in TPS-c and TPS-e/f proteins. Two aspartate-rich motifs, DDxxD and NSE/DTE, are essential for cleaving prenyl diphosphate substrate by chelating a trio of $\mathrm{Mg}^{2+}$ or $\mathrm{Mn}^{2+}$ at the $C$-terminus $[9,11]$. The DDxxD motif was conserved in almost all the DoTPS proteins, except three DoTPS proteins (DoTPS1, DoTPS8 of the TPS-a group, and DoTPS31 of the TPS-c group). DoTPS31 was the only TPS-c member in D. officinale. The TPS-c subfamily is mainly found in land plants and its prenyl diphosphate unit is not cleaved [1]. The NSE/DTE motif was absent in DoTPS8, -11, and -12 of the TPS-a group, DoTPS1, -19, and -24 of the TPS-b group, and DoTPS32 of the TPS-e/f group (Figure 4). Taken together, gene structure and amino acid alignment were consistent with the phylogenetic analysis. The functions of DoTPS proteins in the same group could be inferred from known TPS proteins, according to their phylogenetic relationships. 


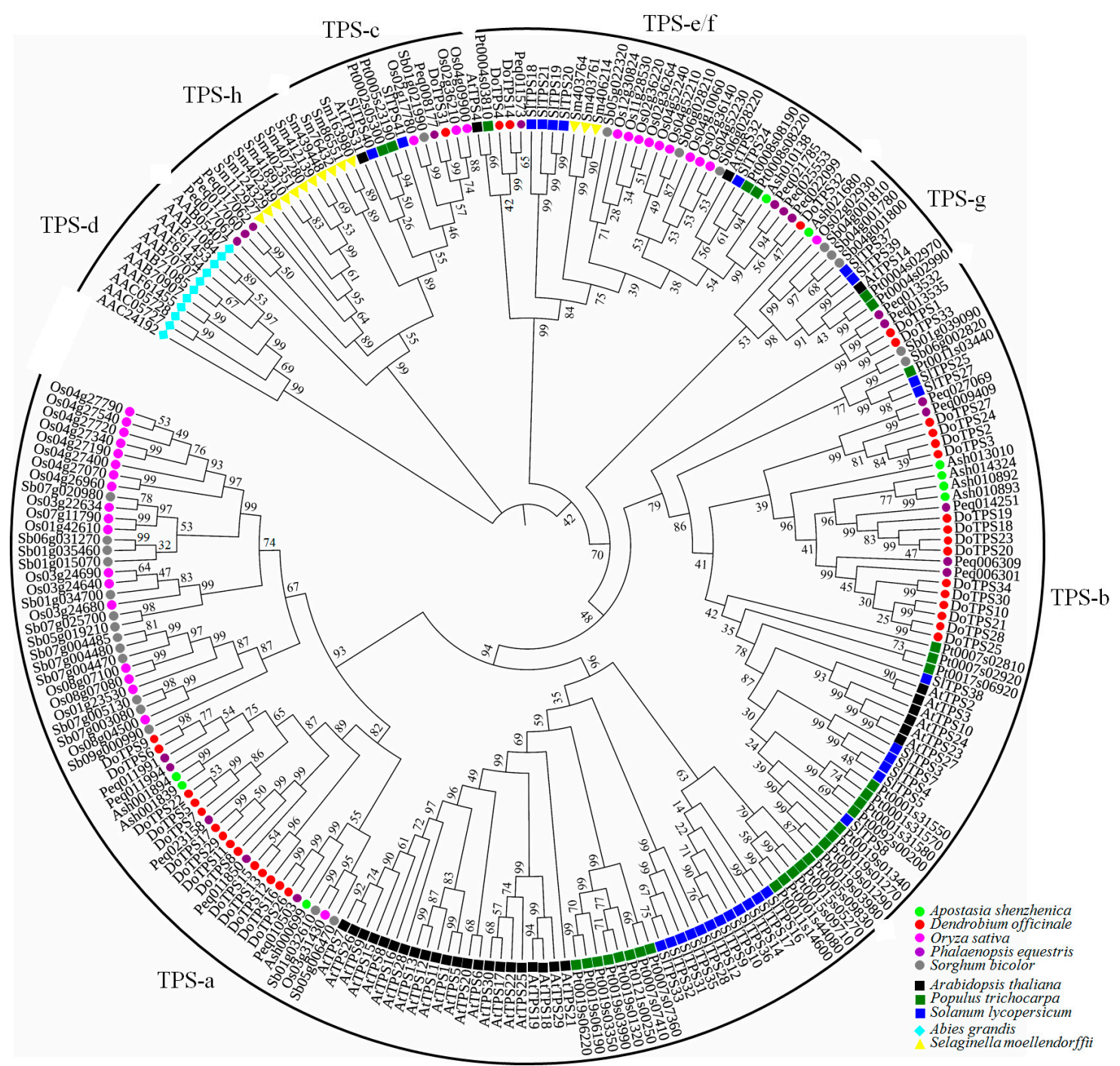

Figure 3. Phylogenetic analysis of TPS proteins in ten higher plant species. A phylogenetic tree was constructed by the neighbor-joining method with the Jones-Taylor-Thornton model and pairwise deletion option using MEGA 7.0 with 1000 bootstrap replicates. Tree visualization and labeling was performed on FigTree v1.4.4 (http://tree.bio.ed.ac.uk/software/figtree/). The TPS family was divided into seven subfamilies as previously reported $[1,15]$ : TPS-a, TPS-b, TPS-c, TPS-d, TPS-e/f, TPS-g, and TPS-h. Circles represented monocotyledonous plants, squares represented dicotyledonous plants, the cyan diamond indicates Abies grandis, and yellow triangle indicates Selaginella moellendorffii. 


\begin{tabular}{|c|c|c|}
\hline & & DDxxD \\
\hline & DoTPS5 & LDEIYET \\
\hline & DoTPS22 & LDEIYDT \\
\hline & DoTPS7 & LDEIYDT \\
\hline & DoTPS6 & LDEIYET \\
\hline & DoTPS9 & LDEIYDS \\
\hline & DoTPS11 & TDEIYDS \\
\hline TPSa & DoTPS29 & TDEIYET \\
\hline $15 \mathrm{Da}$ & DoTPS8 & $\ldots \ldots$ \\
\hline & DoTPS17 & TDEIYDZ \\
\hline & DoTPS13 & LDEIYES \\
\hline & DoTPS2 6 & TDEIYDT \\
\hline & DoTPS16 & TDDIYDZ \\
\hline & DoTPS15 & IDEIYES \\
\hline & DoTPS12 & LDEIYES \\
\hline & DoTPS3 & VDEIYE] \\
\hline & DoTPS2 4 & IDEIYDI \\
\hline & DoTPS2 & VDEIYE] \\
\hline & DoTPS27 & IDEIYD] \\
\hline & DoTPS23 & IDEIYE] \\
\hline & DoTPS18 & IDEIYD] \\
\hline & DoTPS20 & IDEIYD] \\
\hline TPSh & DoTPS21 & IDEVYDS \\
\hline IFSO & DoTPS28 & IDEIYE] \\
\hline & DoTPS25 & MDEIYD] \\
\hline & DoTPS30 & IDEIYE] \\
\hline & DoTPS19 & IDEIYDT \\
\hline & DoTPS34 & IDEIYDI \\
\hline & DoTPS33 & IDEVYDS \\
\hline & DoTPS10 & IDEVYDT \\
\hline & DoTPS1 & PGRSV \\
\hline TPSc & DoTPS31 & VANSTT] \\
\hline & DoTPS4 & ADDFFDF \\
\hline & DoTPS14 & ADDFFDF \\
\hline & DoTPS32 & TDDFFDC \\
\hline
\end{tabular}

\begin{tabular}{|c|c|c|}
\hline & & $\mathrm{RRX}_{8} \mathrm{~W}$ \\
\hline & DoTPS5 & IRDRLIEGYFWV \\
\hline & DoTPS22 & IRDRLIEGCINI \\
\hline & DoTPS7 & IRDRLIEGYIW] \\
\hline & DoTPS6 & SRDRLIECYFWV \\
\hline & DoTPS9 & SRDRLVECYFWV \\
\hline & DoTPS11 & LRDRIVESYFWY \\
\hline $\mathrm{Sa}$ & DoTPS29 & LRDRIVESYFW \\
\hline sa & DoTPS8 & 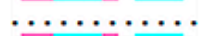 \\
\hline & DoTPS17 & URDRIVESYFWN \\
\hline & DoTPS13 & ARDRLVELNFWY \\
\hline & DoTPS26 & SRDRIVECYFWI \\
\hline & DoTPS16 & SRDRIVECYFWI \\
\hline & DoTPS15 & ARDRFVELHFWY \\
\hline & DoTPS12 & DRDRLVELHFWY \\
\hline & DoTPS3 & AREWPLESYFIA \\
\hline & DoTPS24 & AREWPLESYFIA \\
\hline & DoTPS2 & AREWPVESYFIP \\
\hline & DoTPS27 & ARDWPVESYFLP \\
\hline & DoTPS23 & ARDRLMENYFWI \\
\hline & DoTPS18 & ARDRLMENYFWI \\
\hline & DoTPS20 & ARDRLMENYFWA \\
\hline & DoTPS21 & IRLRLVENHLWP \\
\hline IPSb & DoTPS28 & VRLRLVENYLWZ \\
\hline & DoTPS25 & ARDRLVENYLWI \\
\hline & DoTPS30 & VRDRLVENYIMS \\
\hline & DoTPS19 & ARDRLMETYLWS \\
\hline & DoTPS34 & VRDRLVENYIIS \\
\hline & DoTPS33 & SRLRLMECFFYV \\
\hline & DoTPS10 & IRDRLVENHLWA \\
\hline & DoTPS1 & SRDRLMECFFYV \\
\hline TPSc & DoTPS31 & NQNFVIRAYFIP \\
\hline & DoTPS4 & 3RERTTYCYFAN \\
\hline & DoTPS14 & 3RERTTYCYFAI \\
\hline & DoTPS32 & SRQRETYCYISP \\
\hline
\end{tabular}

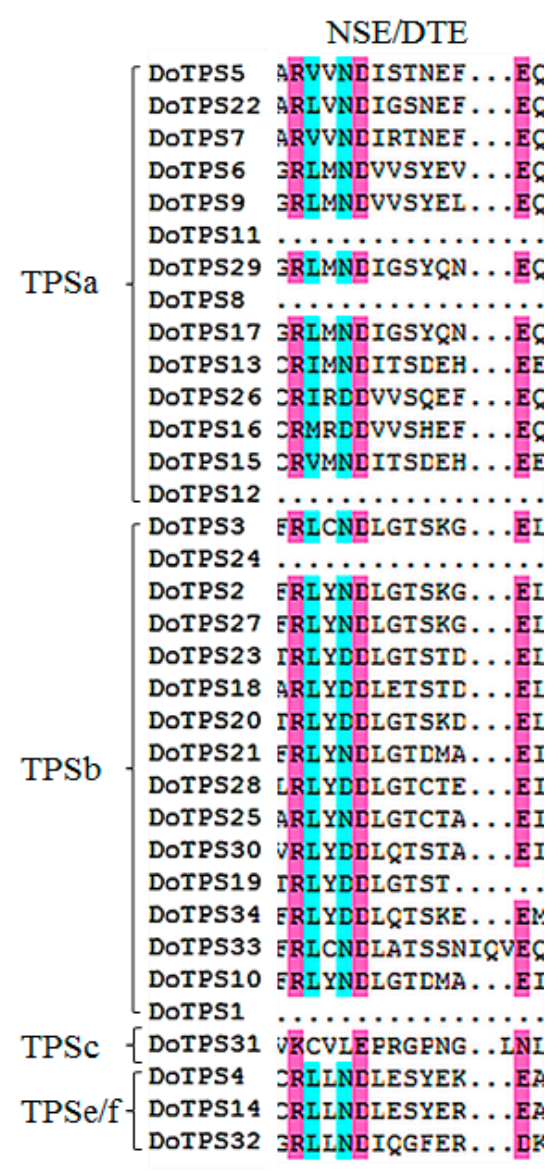

Figure 4. Comparison of DDxxD, $\mathrm{R}(\mathrm{R}) \mathrm{X}_{8} \mathrm{~W}$, and NSE/DTE motifs in D. officinale DoTPS proteins.

\subsection{Identification of Cis-Acting Elements in the Promoter Region of DoTPS Genes}

To ascertain the potential biological roles of DoTPS genes in D. officinale, a 2000-bp upstream region of the initiation code (ATG) was identified using the PlantCARE tool. The cis-acting elements in the promoter regions of DoTPS genes were classified into three categories of cis-elements linked to plant growth and development, phytohormone responsiveness, and stress responsiveness (Figure 5). In the plant growth and development category (159/759), 10 cis-elements involved in circadian rhythms, endosperm expression (AAGAA-motif and GCN4-motif), flowering (AT-rich element, CCAAT-box, and MRE), shoot and root meristem expression (CAT-box), seed expression (RY-element), shoot expression (As-2 element), and zein metabolism $\left(\mathrm{O}_{2}\right.$ site) were found, the highest proportion being the As-2 element (30\%). In the stress responsiveness category (221/759), various elements related to anaerobic induction (ARE, 19\%), defense and stress (TC-rich repeats, $8 \%$ ), dehydration (DRE, $6 \%$ ), drought-inducibility (MBS, 16\%), low temperature (LTR, 5\%), stress (STRE, 27\%), and wounding (WRE3 and WUN-motif, $9 \%$ and 10\%, respectively) responsiveness were detected. Most of cis-elements (379/759) were related to the phytohormone responsiveness category, and were responsive to abscisic acid (ABRE), auxin (TGA-element), ethylene (ERE), gibberellin (GARE-motif, P-box, and TATC-box), MeJA (TGACG-motif and MYC), and salicylic acid (TCA-element). Notably, the largest number of cis-elements was the TGACG-motif and MYC associated with MeJA-responsiveness, accounting for $12 \%$ and $29 \%$ of the hormone-related cis-elements, respectively (Figure 5). These results suggest that DoTPS genes might be MeJA-induced and/or -repressed genes, and that they respond to multiple abiotic stresses. 


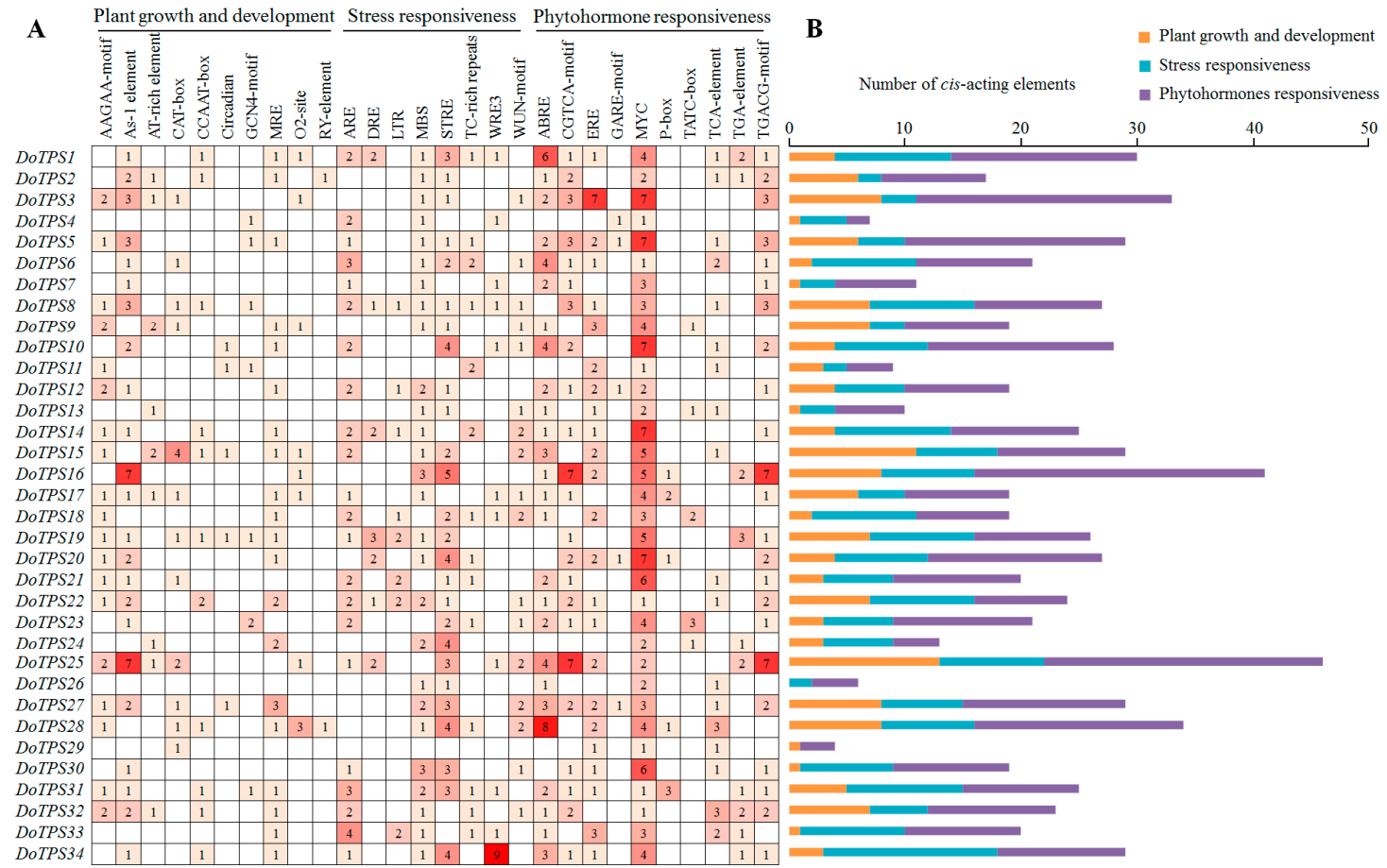

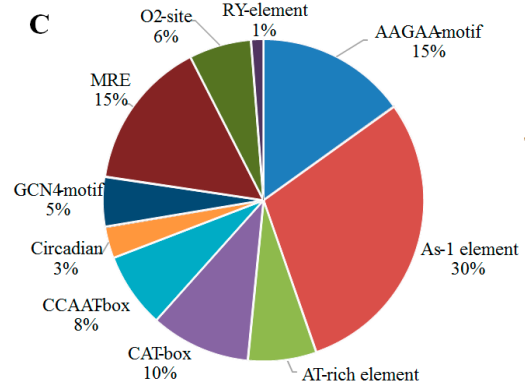

Plant growth and development

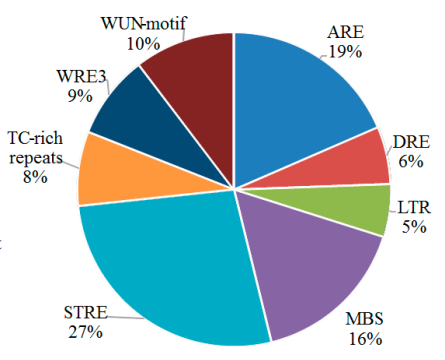

Stress responsiveness

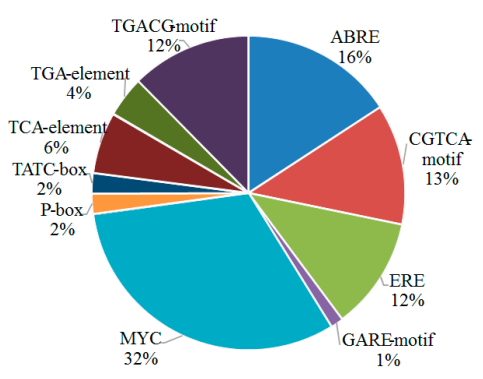

Phytohormone responsiveness

Figure 5. Information of cis-acting elements in DoTPS genes of D. officinale. (A) The gradient red colors and numbers in the grid indicate the number of different cis-acting elements in DoTPS genes. (B) The different colored histogram indicates the number of cis-acting elements in each category. (C) The ratio of different cis-acting elements in each category is shown as pie charts.

\subsection{Tempospatial Expression Patterns of DoTPS Genes in Different D. officinale Organs}

To obtain clues about the role of DoTPS genes in D. officinale development, an RNA-sequencing transcriptome database of flower buds, green root tips, gynostemium (column), labellum (lip), leaves, pollinia, sepals, stems, roots, and white part of roots was established (Figure 6A). Overall, DoTPS genes exhibited distinct organ-specific expression profiles, possibly suggesting the functional divergence of DoTPS genes in different $D$. officinale tissues during growth and development. DoTPS11, -17 , and -19 were highly expressed in stems. Thirteen DoTPS genes exhibited a high level of expression in root tissues, including five (DoTPS4, $-6,-12,-15$, and -29), five (DoTPS13, $-14,-18,-25$, and -32), and three (DoTPS9, -30, and -34) genes in green root tips, roots, and white part roots, respectively. Notably, $52.9 \%$ of DoTPS genes displayed the highest transcript abundance in floral organs. Among them, DoTPS28 in flower buds, DoTPS8 in gynostemium, DoTPS5, -7, -10, -20, -21, -22, and -23 in labellum, DoTPS16, -26, and -31 in pollinium, and DoTPS1, $-2,-3,-24,-27$, and -33 in sepals, indicating that the preferentially expressed DoTPS genes might be indirectly or directly involved in the development of reproductive organs. Our data indicates that the organ-specific expression of DoTPS genes might be important in D. officinale flower growth and development. 

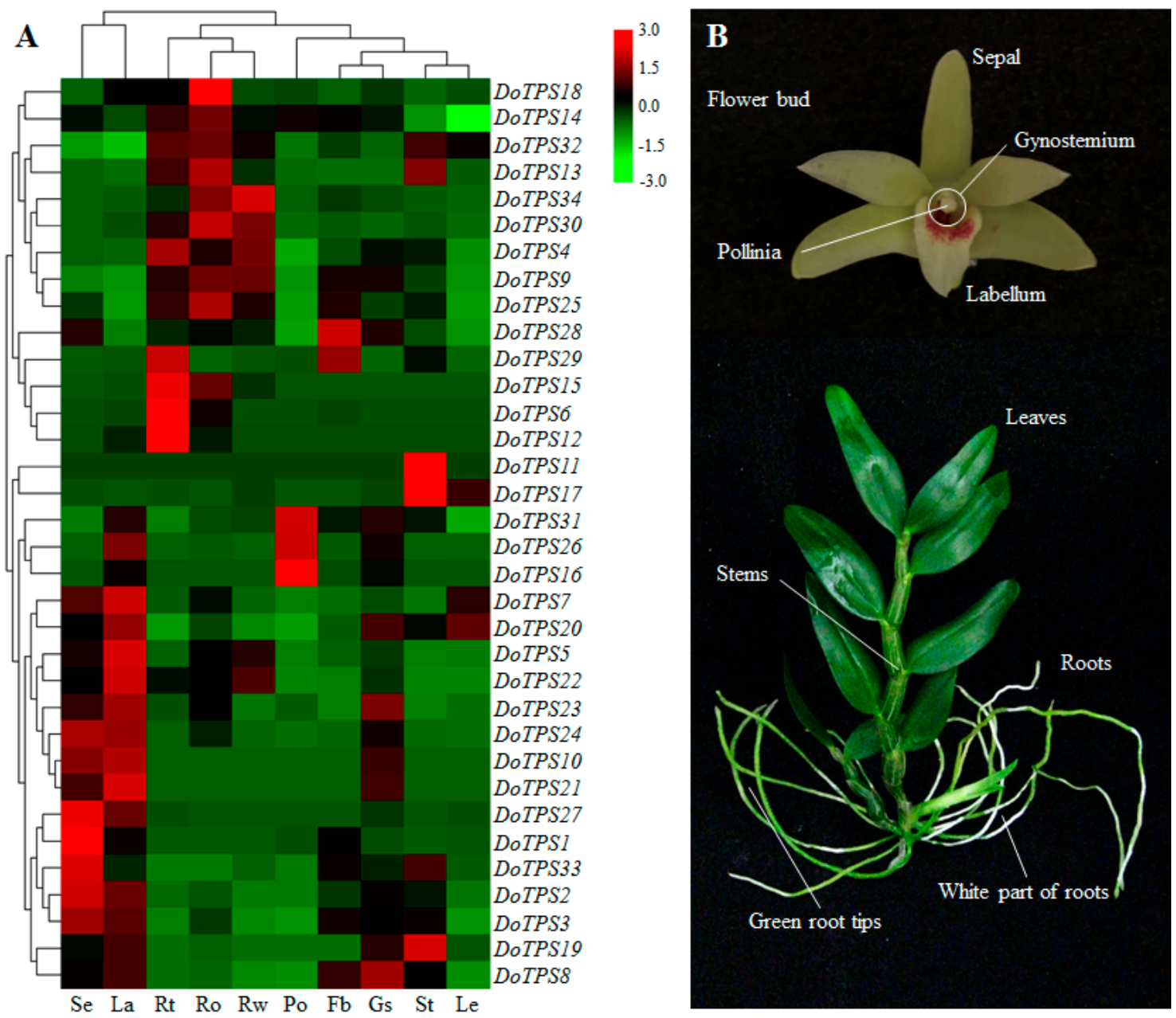

Figure 6. Tissue-specific expression profiles of DoTPS genes in different D. officinale organs. (A) The transcription levels of DoTPS genes in different tissues. The different tissues were sepals (Se), labellum (la), green root tips (Rt), roots (Ro), white part of roots $(\mathrm{Rw})$, pollinia $(\mathrm{Po})$, flower buds $(\mathrm{Fb})$, gynostemium (Gs), stems (St), and leaves (Le) in two-year-old D. officinale adult plants. Heatmap was generated using the TBtools server (https://github.com/CJ-Chen/TBtools), and gradient color from green to red was expressed as the log2-transformed expression levels of each DoTPS gene that was normalized to the internal reference gene DoEF-1 $\alpha$, GenBank accession no. JF825419. (B) D. officinale "Zhongke 5" used in this study. All fragments per kilobase of transcript per million fragments mapped (FPKM) values that were used were downloaded from NCBI under BioProject PRJNA262478 [25], and are listed in Table S7.

\subsection{Expression Patterns of DoTPS Genes under Abiotic Stress}

To better understand the role of DoTPS genes in response to cold and osmotic stresses, transcriptome data combined with the RT-qPCR assay were employed to investigate the expression levels of DoTPS genes in D. officinale under cold $\left(0^{\circ} \mathrm{C}\right)$ or osmotic (mannitol) treatment. Results showed that DoTPS genes exhibited distinct expression patterns under osmotic treatment, showing two trends, an upward trend and a downward trend (Figure 7A). Half of the DoTPS genes, including DoTPS1, $-3,-6,-8,-11$, $-16,-18,-19,-21,-22,-23,-24,-25,-26,-27,-31$, and -32 , were obviously suppressed (1.2-48.7-fold) by mannitol-induced osmotic stress. The other half of DoTPS genes exhibited an increasing trend, but the highest expression level was either at $12 \mathrm{~h}$ (DoTPS9, -10, -14, and -28), $24 \mathrm{~h}$ (DoTPS2, -4, -7, -12, -13, -15, $-20,-29$, and -30), or $48 \mathrm{~h}$ (DoTPS5, -17, -33, and -34) in response to mannitol treatment (Figure 7A).

After cold acclimation $\left(0^{\circ} \mathrm{C}\right)$ for $20 \mathrm{~h}, 26$ DoTPS genes were upregulated, more than the number of suppressed genes (8; Figure $7 \mathrm{~B})$. Compared to the non-acclimated controls, the transcription levels of DoTPS4, -8, -13, -15, -23, -26, and -33 were downregulated between 1.6- and 14.3-fold. In contrast, 
the expression levels of most other DoTPS genes (27/34) were clearly upregulated between 1.9- and 103.1-fold, except for the tiny variation of DoTPS20 and DoTPS32. These results suggested that DoTPS genes might be involved in cold and osmotic stress responses in $D$. officinale.

A

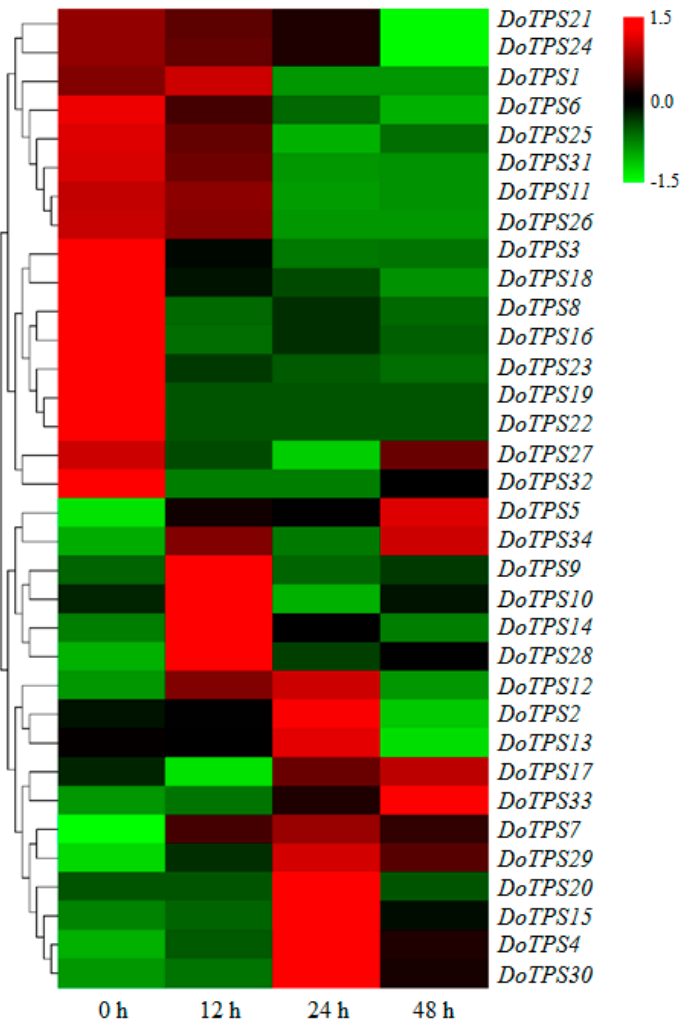

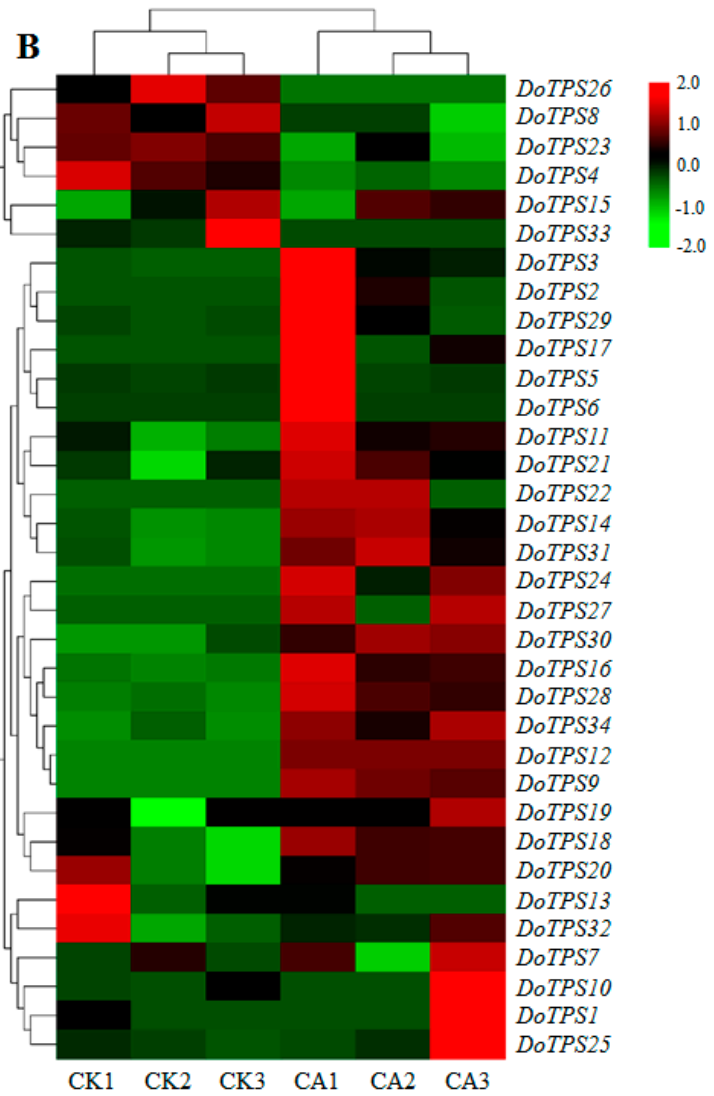

CK1 CK2 CK3 CA1 CA2 CA3

Figure 7. Transcription levels of DoTPS genes in D. officinale under cold and osmotic stresses. (A) Expression profiles of DoTPS genes in response to $200 \mathrm{mM}$ mannitol treatment for $48 \mathrm{~h}$. (B) Expression profiles of DoTPS genes in response to cold treatment $\left(0^{\circ} \mathrm{C}\right)$ for $20 \mathrm{~h}$. Heatmap was drawn using TBtools software (https://github.com/CJ-Chen/TBtools). Color gradient from green to red was expressed as the log2-transformed expression level of each DoTPS gene. CA, cold acclimation; CK, control (non-acclimation). The expression values of DoTPS genes in response to mannitol treatment are listed in Table S8. The FPKM values of DoTPS genes exposed to cold treatment that were downloaded from a transcriptome database [29], are listed in Table S9.

\subsection{Expression Patterns of DoTPS Genes Subjected to MeJA Treatment}

MeJA is a signaling molecule that promotes the formation of secondary metabolic products [26]. We determined the effect of MeJA at the level of transcription of DoTPS genes in D. officinale. MeJA treatment differentially regulated DoTPS gene expression. Compared to the non-treated control, DoTPS28, -31, and -32 were suppressed between 1.5- and 2.4-fold at $12 \mathrm{~h}$ after MeJA treatment. The suppressed genes returned to their control level at $48 \mathrm{~h}$ after MeJA treatment. In contrast, 31 DoTPS genes were upregulated between 1.2- and 45.1-fold, with the highest expression at $12 \mathrm{~h}$ (21/31), $24 \mathrm{~h}$ (8/31), or $48 \mathrm{~h}(2 / 31)$ after MeJA treatment (Figure $8 \mathrm{~A})$, but others showed reduced gene expression. Terpenes are dominant floral volatiles of orchids, especially geraniol and linalool [7,24]. Moreover, after treatment with MeJA, the amount of geraniol and linalool was significantly enhanced (Figure 8B). DoTPS genes were inducible by MeJA, which might be related to the cis-acting elements present in their promoters, resulting in the increased formation of terpenes in D. officinale. 
A

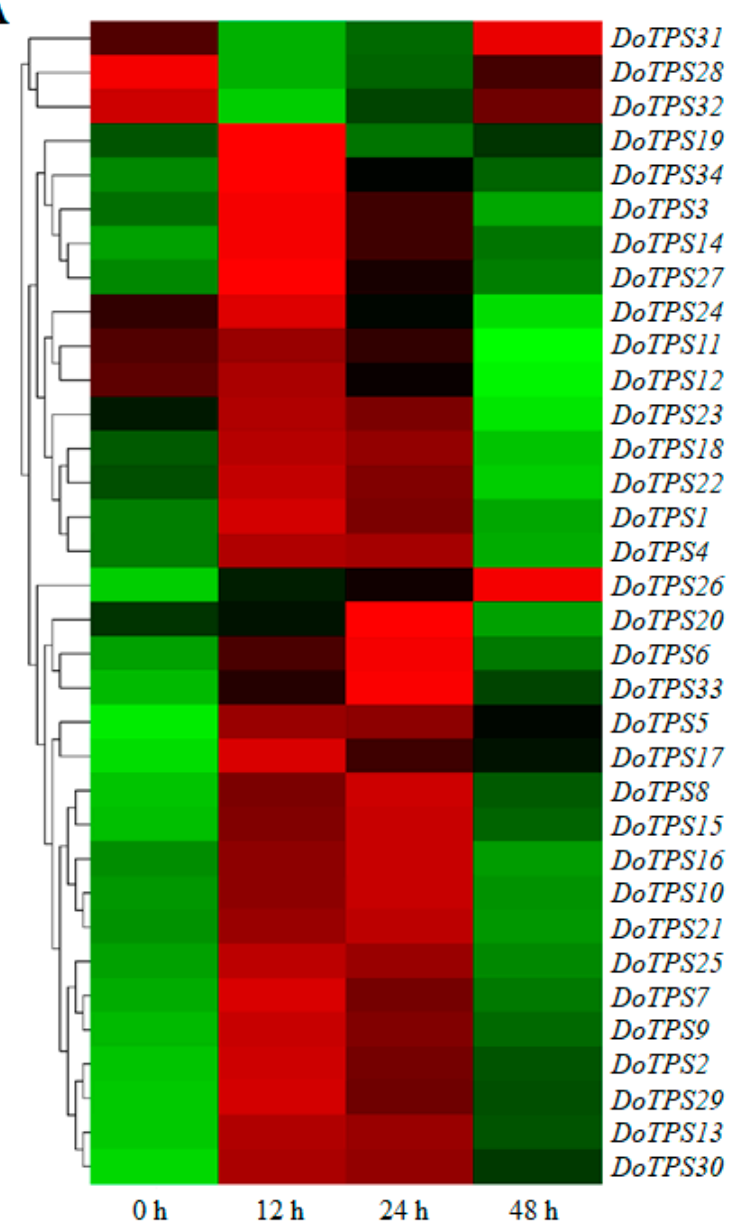

B
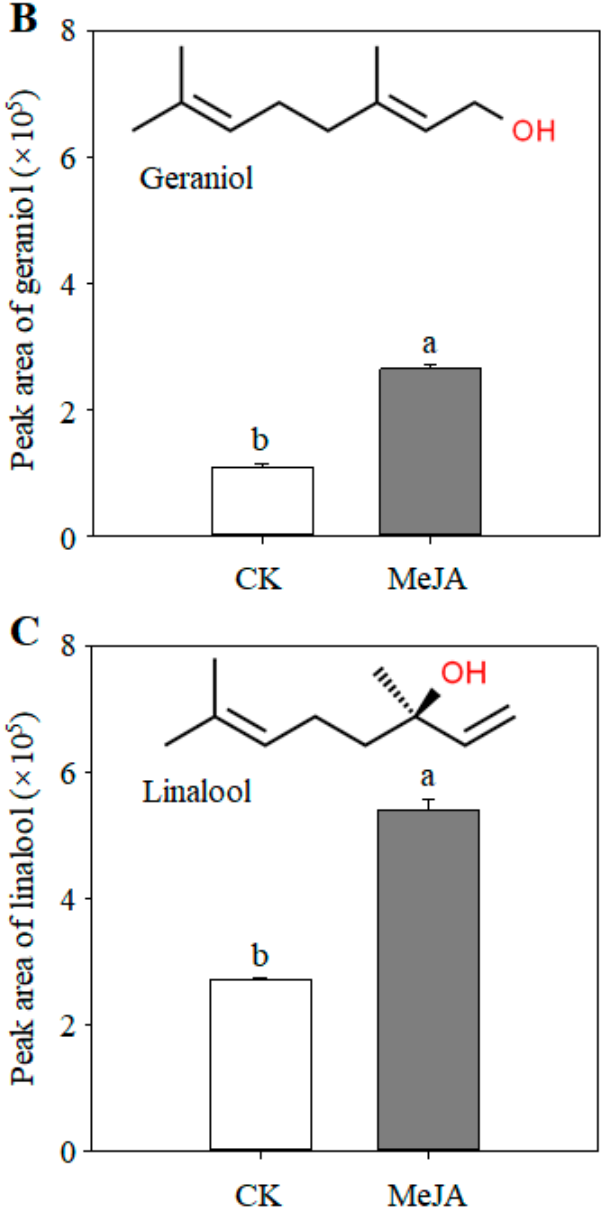

Figure 8. The transcription levels of DoTPS genes and synthesis of geraniol and linalool in D. officinale after MeJA treatment. (A) Effect of MeJA treatment on the expression of DoTPS genes. (B) Effect of MeJA treatment on the synthesis of geraniol. (C) Effect of MeJA treatment on the synthesis of linalool. Ten 10-month-old D. officinale seedlings exposed to $1 \mathrm{mM}$ MeJA for $0,12,24$, and $48 \mathrm{~h}$ were harvested. Heatmap was created using the TBtools software (https:/github.com/CJ-Chen/TBtools), and gradient color from green to red was expressed as the log2-transformed expression levels of each DoTPS gene. Each bar represents the mean ( \pm standard error, $n=3$ ) of three independent biological replicates. Different letters above the bars indicated significant differences $(p<0.05$, Duncan's multiple range test). CK, control treatment without MeJA. MeJA, methyl jasmonate. The expression values of DoTPS genes in response to MeJA treatment are listed in Table S10.

\subsection{Transcription Abundance of DoTPS Genes at Budding and Flowering Stages}

Since the majority of DoTPS genes were highly expressed in floral organs (Figure 6), we further investigated their spatial expression patterns at three floral developmental stages. DoTPS6, $-7,-9,-11$, $-16,-18,-21,-22,-23,-26,-28,-30,-31,-32$, and -34 were mainly expressed in the floral budding stage. DoTPS4, $-5,-8,-10,-15,-19,-20$, and -25 were prominently expressed during the semi-flowering stage. The remaining genes (DoTPS1, $-2,-3,-12,-13,-14,-17,-24,-27,-29$, and -33) displayed greatest expression levels at the full flowering stage (Figure 9). DoTPS genes responsible for floral fragrance showed significant differences among the three floral developmental stages. Notably, DoTPS10 had the highest level of transcription during the floral developmental stages (Figure 9), suggesting that DoTPS10 may be responsible for the biosynthesis of geraniol or linalool. Further functional characterization of the DoTPS10 gene could be helpful. 

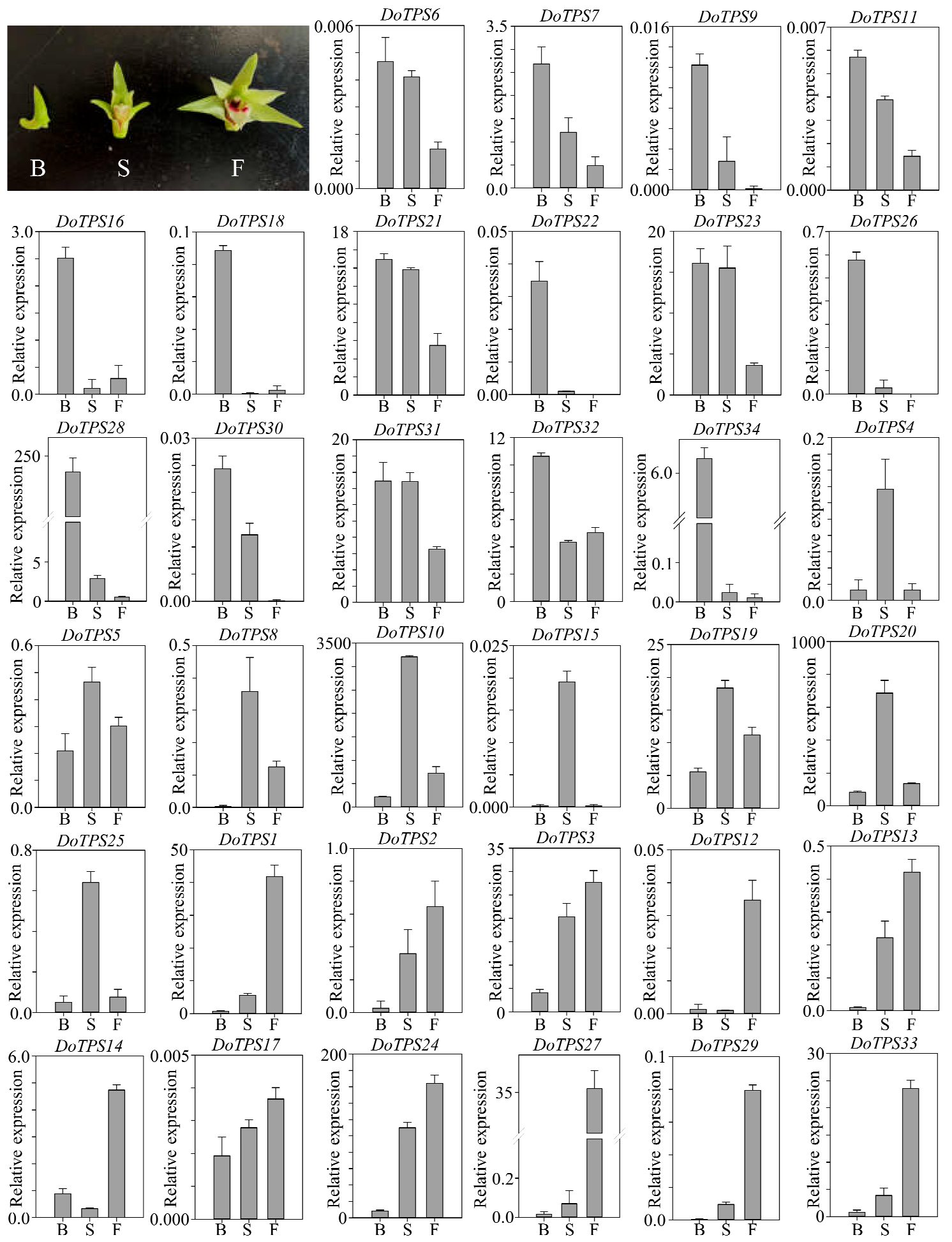

Figure 9. Transcription levels of DoTPS genes in D. officinale at three flowering stages: budding (B), semi-flowering (S) and full flowering (F). The levels of transcription were calculated by the $2^{-\Delta \Delta C T}$ method and normalized to the $\mathrm{C}_{\mathrm{T}}$ value of $D o E F-1 \alpha$. Each bar represents the mean ( \pm standard error, $n=3$ ) of three independent biological replicates. Different letters above bars indicate significant differences ( $p<0.05$, Duncan's multiple range test). The expression values of DoTPS genes at three flowering stages of D. officinale are listed in Table S11. 


\subsection{Subcellular Localization of DoTPS10 in Heterologous Plants}

DoTPS10 was assigned to the chloroplast with a 46 aa transit peptide. To validate the prediction, YFP-tagged DoTPS10 fusions were transiently expressed in A. thatiana protoplasts. In vivo YFP fluorescence signals from DoTPS10 were observed in chloroplasts (Figure 10A-D), which were consistent with a previous report of FhTPS1, FhTPS2, FhTPS4, and FhTPS5 in a Freesia hybrid [21].
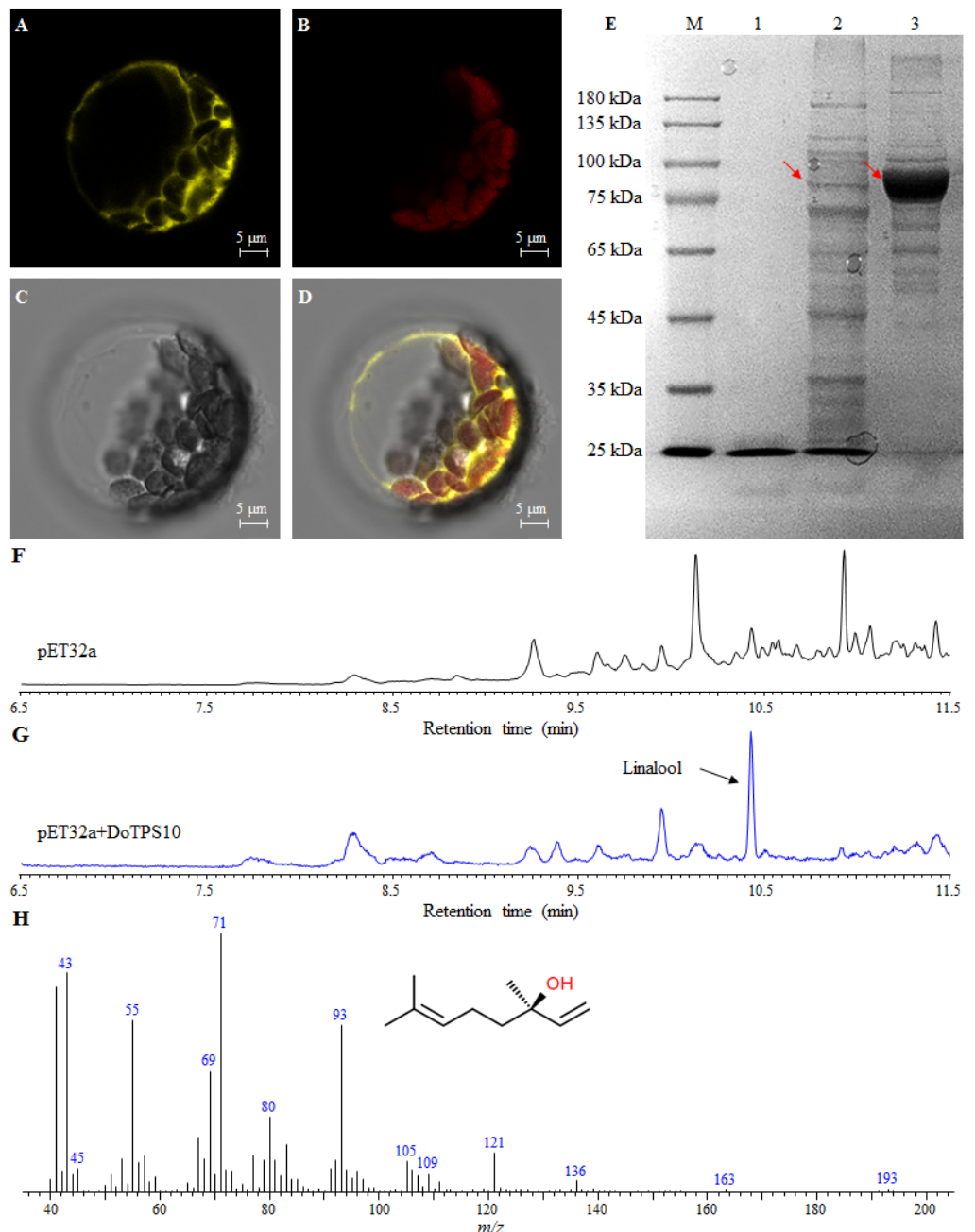

Figure 10. Functional characterization of DoTPS10. (A-D) Subcellular localization of DoTPS10 fused with yellow fluorescent protein (YFP) and transiently expressed in A. thaliana protoplasts. Bars = $5 \mu \mathrm{m}$. (E) SDS-PAGE analysis of DoTPS10 recombinant protein expressed in Escherichia coli BL21. Lanes M, 1, 2, and 3 indicate marker, pET32a, crude DoTPS10 protein, and purified DoTPS10 protein, respectively. Red arrow indicates target protein. (F-G) Gas chromatograms of products yielded by DoTPS10 using GPP as a substrate. (H) Mass spectrum of linalool was identical to the mass spectrum of the linalool standard.

\subsection{Functional Characterization of DoTPS10 Involved in the Formation of Linalool}

Generally, monoterpene volatiles are the main terpenes in orchids. These are produced by TPS proteins using GPP as a substrate [6,7]. To further confirm the role of DoTPS10 in the synthesis of monoterpenes, a His-tagged vector (pET32a) was used to produce the recombinant DoTPS10 protein. The vector was successfully expressed as a soluble protein in E. coli BL21. DoTPS10 protein was purified using a His-trap Ni-sepharose high performance column. His-tagged vector (pET32a) had an Mw of $23.8 \mathrm{kDa}$, containing $4.8 \mathrm{kDa}$ of six His-tags, and recombinant DoTPS10 protein exhibited an approximate Mw of $88.3 \mathrm{kDa}$ on SDS-PAGE (Figure 10E). After recombinant DoTPS10 protein 
was incubated with GPP, gas chromatography-mass spectrometry (GC-MS) analysis showed that empty pET32a could not produce linalool while the recombinant DoTPS10 protein singly converted the substrate GPP to the corresponding product linalool (Figure S3, Figure 9G,H).

\section{Discussion}

D. officinale is widely grown in subtropical and temperate regions and used as a health food, but also has a high ornamental and medicinal value [22,30]. Epiphytic or lithophytic herbs commonly suffer from adverse environmental conditions such as chilling, drought, and water deficit [22,23,29-32]. Plant volatile terpenes play critical roles not only in the formation of orchid floral scents, but also in response to environmental stresses [1,2,5-7]. TPS is the primary enzyme responsible for catalyzing the formation of monoterpenes $\left(C_{10}\right)$, sesquiterpenes $\left(C_{15}\right)$, or diterpenes $\left(C_{20}\right)$ from the substrates GPP, FPP, or GGPP, respectively (Figure 1). Therefore, studies on floral scents have mainly focused on the identification and analysis of TPS genes responsible for the biosynthesis of terpenes [7,24].

Herein, 34 DoTPS genes were identified in the D. officinale genome according to the conserved C-terminal and N-terminal domain of TPS, followed by manual verification (Table 1; Figure 3). TPS subfamilies belong to a medium-sized family, with various gene numbers (approximately 20-150) among different plant species $[1,10]$. For example, 32 AtTPS genes were functionally discovered in A. thaliana [11]. A total of 14 SmTPS, 33 OsTPS, and 152 VvTPS genes were found in S. moellendorffii, $O$. sativa, and $V$. vinifera, respectively $[1,10,17,18]$. Furthermore, TPS occupied 0.26 genes/M in the A. thaliana genome (125 M) [33], 0.13 genes/M in S. moellendorffii (106 M) [34], 0.08 genes/M in the O. sativa genome ( $389 \mathrm{M}$ ) [35], 0.31 genes/ $\mathrm{M}$ in $V$. vinifera $(487 \mathrm{Mb}$ ) [36], and 0.02 genes/M in $D$. officinale (1.35 G) [22-24]. It is possible that tandem duplication may have occurred during evolution of the D. officinale genome, mainly in the TPS-a and TPS-b subfamilies.

Phylogenetic analysis showed that DoTPS proteins fall into four known angiosperm TPS subfamilies (TPS-a, TPS-b, TPS-c, and TPS-e/f), with the exception of the gymnosperm-specific TPS-d and S. moellendorffii-specific TPS-h (Figure 3). TPS-b was the largest subfamily among the DoTPS proteins, followed by TPS-a, which was consistent with Daucus carota [14], but in contrast to other species such as A. thaliana (22 of the 32 TPS genes were TPS-a genes) [12] and S. lycopersicum (12 of the 29 TPS genes were TPS- $a$ genes) [17]. As illustrated in Table S12, 16 DoTPS proteins were annotated as monoterpene synthase, Fourteen DoTPS proteins were annotated as sesquiterpene synthase, and four DoTPS proteins were annotated as diterpene synthase. These four putative diterpene synthases harbored one TPS-c and three TPS-e/f proteins. TPS-e/f proteins can produce mono-, sesqui-, and di-terpenes [1]. All 14 sesquiterpene synthases and 16 monoterpene synthases were assigned to TPS-a and TPS-b, respectively (Figure 4; Table S12). For mono- and sesquiterpenes, subcellular location and availability of substrate are more important for the characterization of typical products produced in vivo. FhTPS6 was localized in the cytosol, and was deemed to be associated with the formation of a sesquiterpene (nerolidol) and several monoterpenes (myrcene, limonene, cis-ocimene, trans-ocimene, and terpinolene), suggesting that GPP and FPP might move from plastids to the cytosol [21]. TPS-a can produce monoterpenes in vitro, while TPS-b can produce hemi-, mono-, and sesquiterpenes in vitro [1]. TPS-a is an angiosperm-specific clade that is responsible for sesquiterpene or diterpene synthases, and can be further divided into monocotyledonous- and dicotyledonous-specific subgroups $[1,9,10]$. In A thaliana, four TPS-a genes encode cytosolic sesquiterpene synthases, while the other nine TPS- $a$ genes harbored transit peptides presumably encoding diterpene synthases, although their functions have not yet been fully investigated [12]. In S. lycopersicum, all TPS-a genes only encode sesquiterpene synthases, and 11 of 12 are cytosolic and not chloroplastic [17]. Similarly, most proteins previously functionally identified from the angiosperm-specific TPS-b clade are monoterpene synthases. For example, six A. thaliana TPS-b proteins clustered in the same branch that harbored AtTPS10, a monoterpene synthase that produces myrcene or ocimene [12]. These findings indicate that the TPS members share a similar functional feature within the same subfamily, however, further functional characterization is required. 
The expression analysis showed that DoTPS genes were mainly expressed in floral organs of D. officinale, followed by root organs and stems (Figure 6), inferring a strict regulation of terpenoid production. Interestingly, 11 of 18 DoTPS- $b$ genes with high transcript levels in floral organs were monoterpene synthase genes (Figure 6). Four monoterpene synthase genes (DoTPS10, -19, -20, and -25) were highly expressed at the semi-flowering stage (Figure 9), in agreement with the content of geraniol and linalool (Figure S2). Among them, DoTPS10 showed the highest transcript level in floral organs (Figures 6 and 9), suggesting that it may be responsible for the biosynthesis of monoterpenes. In the present study, DoTPS10 was shown to be a single-product enzyme that could covert GPP to linalool (Figure 10), the predominant component of floral scents in orchids. TPS proteins that produce the same single product have also been found in a Freesia hybrid [21], Malus domestica [16] and Vitis vinifera [19]. Furthermore, the majority of DoTPS genes could be induced by MeJA treatment, resulting in the increased production of monoterpene volatiles such as geraniol and linalool (Figure 8). The reason why exogenous MeJA resulted in the upregulation of these DoTPS genes may be due to the presence of G-boxes in their promoters (Table S13), which can interact with the existing CGTCA or MYC motif of the jasmonic acid signaling pathway [37], but it needs to be further explored. Therefore, activated expression of DoTPS10 by MeJA treatment offers a critical cue for further exploring the mechanism of linalool biosynthesis in $D$. officinale.

RT-qPCR data showed that distinct tempospatial expression profiles of DoTPS genes could be affected when D. officinale was exposed to cold or osmotic treatment (Figure 7). Similarly, previous work has emphasized the importance of terpenes in defensive response to biotic attack and abiotic stresses $[1-5,7,9,10]$. It is possible that the crassulacean acid metabolism plant $D$. officinale, which adheres tightly to the surface of tree bark or rocks in locations with limited soil, thus requires TPS proteins to quickly biosynthesize terpenes to circumvent adverse environments $[23,25]$. To better understand the mechanisms of differential terpenoid production in $D$. officinale, more efforts should be made to integrate studies on DoTPS expression patterns with those on profiling of terpenes.

\section{Materials and Methods}

\subsection{Plant Materials}

D. officinale “Zhongke 5" (http://www.cas.cn/syky/201811/t20181109_4669776.shtml, Figure 6B) with better adaptability to adverse habitats was cultivated in a greenhouse and in the open air at the South China Botanical Garden, Chinese Academy of Sciences (Guangzhou, Guangdong Province, China) under natural light and controlled temperatures, between 25 and $30^{\circ} \mathrm{C}$. Flowers, leaves, roots, and stems from 14-month-old adult plants of full-sib D. officinale were sampled at the flowering stage. For osmotic treatment, 10 independent 10-month-old D. officinale seedlings were transferred to fresh half-strength Murashige and Skoog (MS) medium [38] supplemented with $200 \mathrm{mM}$ mannitol. Control seedlings were transferred in same way without additional mannitol. For the MeJA treatment, the same seedlings were transferred to fresh half-strength MS medium supplemented with $1 \mathrm{mM}$ MeJA, and MeJA-free medium was used as the control. The leaves from osmotic and MeJA treatments were collected after treatment at $0,12,24$, and $48 \mathrm{~h}$. All samples were frozen immediately in liquid nitrogen, and stored at $-80^{\circ} \mathrm{C}$ until use.

\subsection{Identification of TPS Family Members in D. officinale}

The recently released D. officinale genome [23] was used in this study. Two specific TPS domains, PF03936 and PF01397, which respectively indicate the $C$-terminal and $N$-terminal domain of TPS from the Pfam database (http://pfam.xfam.org/), were used to build the corresponding Hidden Markov Model (HMM) file. HMMER v3.3 (http://www.hmmer.org/) was used to search the D. officinale protein database with the PF03936 and PF01397 domains model data as queries. Significant hits (e-value $<10^{-3}$ ) were retrieved as candidate $D$. officinale TPS proteins. To verify the sequences, BLASTp (http://blast.ncbi.nlm.nih.gov) and SMART (http://smart.embl-heidelberg.de/) searches of the 
retrieved TPS proteins were carried out. Non-redundant sequences that did not contain the terpene synthase $C$-terminal domain and terpene synthase $N$-terminal domain were removed. The grand average of hydrophobicity (GRAVY), molecular weight (Mw), and isoelectric points (pI) of the TPS proteins were predicted from the ExPASy database (http://expasy.org/). The aliphatic index (AI), and instability index (II) were calculated by EMBOSS Pepstats tool (https://www.ebi.ac.uk/Tools/). Plant-mPLoc (www.csbio.sjtu.edu.cn/bioinf/plant-multi/), AtSubP (http://bioinfo3.noble.org/AtSubP/), and pLoc-mPlant (www.jci-bioinfo.cn/pLoc-mPlant/) were used to predict the subcellular localization of TPS proteins. The secondary structure of TPS proteins was determined using the SOPMA program (http://npsa-pbil.ibcp.fr/). In addition, TPS proteins from P. equestris and A. shenzhenica were obtained from their reported genome database [25]. The other TPS proteins from A. grandis, A. thaliana, O. sativa, P. trichocarpa, S. moellendorffii, S. lycopersicum, and S. bicolor were downloaded from the Phytozome version 12.1 database (https://www.phytozome.net).

\subsection{Conserved Motifs, Gene Structure, and Phylogenetic Analysis}

Conserved motifs of TPS proteins were analyzed with MEME software (http://meme-suite.org/) with default parameters. The exon-intron structure of TPS proteins was aligned with the Gene Structure Display Server (GSDS 2.0, http://gsds.cbi.pku.edu.cn/). Multiple sequence alignment was performed using TPS proteins from A. grandis, A. shenzhenica, A. thaliana, D. officinale, O. sativa, P. equestris, P. trichocarpa, S. moellendorffii, S. lycopersicum, and S. bicolor with ClustalX 2.1 software (www.clustal.org/). The alignments were manually adjusted and truncated with a focus on diagnostically conserved regions such as the DDxxD, NSE/DTE, and $\mathrm{RRX}_{8} \mathrm{~W}$ motifs, based on a reported protocol [15]. A phylogenetic tree was constructed using the neighbor-joining (NJ) method [39] under the Jones-Taylor-Thornton (JTT) model with 1000 bootstrap replicates in MEGA version 7.0 [40]. The generated graph was redrawn and annotated by Figtree version 1.4.4 (http://tree.bio.ed.ac.uk/software/figtree/). The sequences of TPS proteins used in this study can be found in Table S6.

\subsection{Total RNA Isolation, cDNA Reverse Transcription, and RT-qPCR Analysis}

Total RNA from the flowers and leaves of 14-month-old D. officinale "Zhongke 5" at the flowering stage were extracted using the Quick RNA Isolation Kit (Huayueyang, Beijing, China) according to the instruction manual. Genomic DNA contamination was eliminated with RNase-free DNase I (TaKaRa, Dalian, China). First-strand cDNA was synthesized by reverse transcription with the help of the PrimeScript ${ }^{\mathrm{TM}}$ RT Reagent Kit (Takara) according to the manufacturer's protocol. SYBR ${ }^{\circledR}$ Premix Ex Taq ${ }^{\mathrm{TM}}$ (TaKaRa) was applied for RT-qPCR analysis on a LightCycler 480 instrument (Roche Diagnostics, Mannheim, Germany) as described previously [41]. D. officinale elongation factor 1- $\alpha$ (DoEF-1 $\alpha$, GenBank accession no. JF825419) was selected as the internal reference gene [42]. At least three biological replicates were carried out, and relative mRNA expression data were quantified by the $2^{-\triangle \Delta C T}$ method [43]. The RT-qPCR primers of TPS genes listed in Table S14 were acquired by the PrimerQuest tool (http://www.idtdna.com/Primerquest/Home/Index).

\subsection{Cis-Acting Elements Analysis of TPS Genes in D. officinale}

The promoter sequences, $2000 \mathrm{bp}$ upstream of the translational start site (ATG), of TPS genes in D. officinale were obtained from the D. officinale genome [23]. Afterwards, the online software PlantCARE (http://bioinformatics.psb.ugent.be/webtools/plantcare/html/) was employed to investigate putative cis-regulatory elements in the promoter region of DoTPS genes in D. officinale.

\subsection{Gene Expression Analysis Based on Transcriptome Data}

To gain insight into the tissue-specific transcription levels of DoTPS family genes, raw data from the RNA-sequencing of 10 different tissues (i.e., flower buds, green root tips, gynostemium (column), labellum (lip), leaves, pollinia, sepals, stems, roots, and the white part of roots) in two-year-old D. officinale adult plants was downloaded from NCBI under BioProject PRJNA262478 [25]. To study the 
effects of cold acclimation $\left(0^{\circ} \mathrm{C}\right.$ for $\left.20 \mathrm{~h}, \mathrm{CA}\right)$ and non-acclimation $\left(20^{\circ} \mathrm{C}\right.$ for $\left.20 \mathrm{~h}, \mathrm{CK}\right)$ on DoTPS gene expression, the raw RNA-sequencing reads were retrieved from a reported transcriptome database [29]. Fragments per kilobase of transcript per million mapped reads (FPKM) values of DoTPS genes in tested samples were used to evaluate transcription abundance. DoEF-1 $\alpha$ was selected as the internal reference gene for normalizing each expression value. The heat maps of the DoTPS genes' expression patterns were illustrated using the TBtools software with default settings (https://github.com/CJ-Chen/TBtools), and the gradient color from green to red is expressed as the log2-transformed expression levels of each DoTPS gene.

\subsection{Gas Chromatography-Mass Spectrometry Analysis of Geraniol and Linalool in Flowers of D. officinale}

The frozen flowers of D. officinale (500 mg) were ground to a fine powder in liquid nitrogen, and then blended with precooled dichloromethane $(3 \mathrm{~mL}$ ) by vortexing for $2 \mathrm{~min}$, followed by shaking at $25^{\circ} \mathrm{C}$ for $8 \mathrm{~h}$ in the dark. The supernatant was collected by $13,000 \times g$ centrifugation, and concentrated to $200 \mu \mathrm{L}$ using a stream of $\mathrm{N}_{2}$ before analysis by gas chromatography-mass spectrometry (GC-MS, Shimadzu Co., Kyoto, Japan) equipped with a 30-m Supelcowax-10 column (0.25 mm diameter $\times$ $0.25 \mu \mathrm{m}$ film thickness). The temperature program was isothermal at $60^{\circ} \mathrm{C}$ for $3 \mathrm{~min}$, then increased at a rate of $4{ }^{\circ} \mathrm{C} \mathrm{min}-1$ to $240{ }^{\circ} \mathrm{C}$, and maintained at $240{ }^{\circ} \mathrm{C}$ for $20 \mathrm{~min}$. MS analyses were performed in full-scan mode with a mass range from $\mathrm{m} / \mathrm{z} 40$ to 200 . Geraniol and linalool were identified against the NIST 2008 mass spectra library (https://chemdata.nist.gov/) as described previously [44].

\subsection{Prokaryotic Expression and DoTPS10 Enzyme Assay in Escherichia coli}

The full-length DoTPS10 was amplified from first-strand cDNA, as published previously [41]. The obtained PCR product was purified and inserted into the pMD-18T vector (TaKaRa) for sequencing. The gene-specific primers used for DoTPS10 are indicated in Table S14.

A 1797-bp ORF without a stop codon (TAA) of DoTPS10 was cloned into the pET32a vector with SalI and XhoI restriction sites. DoTPS10 expression in E. coli BL21 (DE3) cells and purification using a His-trap Ni-sepharose high performance column (GE Healthcare, Fairfield, CT, USA) were described in our previous study [45]. The purified pET32a-DoTPS10 protein was fractionated by $10 \%$ sodium dodecyl sulfate polyacrylamide gel electrophoresis (SDS-PAGE).

In vitro DoTPS10 enzyme assays were performed in screw-cap 5-mL glass vials containing $1 \mathrm{~mL}$ of 2-hydroxy-3-morpholinopropanesulfonic acid (MOPSO) buffer (10 mM, pH 7.0, containing $5 \mathrm{mM}$ dithiothreitol, $10 \mathrm{mM} \mathrm{MgCl}$, and $10 \mathrm{mM}$ GPP as substrate) and $100 \mu \mathrm{g}$ of DoTPS10 protein. The reactions were overlaid with $200 \mu \mathrm{L}$ of $n$-hexane and incubated at $30^{\circ} \mathrm{C}$ for $1 \mathrm{~h}$. The mixtures were mixed vigorously for $1 \mathrm{~min}$ to obtain the enzymatic products. The organic phase was removed, and $1 \mu \mathrm{L}$ was detected using GC-MS as described above. For comparison, His-tagged protein (empty pET32a) was used as the blank control.

\subsection{Subcellular Localization of DoTPS10 in A. thaliana Mesophyll Protoplasts}

To determine the localization of DoTPS10, a 1797-bp ORF without a stop codon (TAA) of DoTPS10 was introduced into the pSAT6-EYFP-N1 vector with an NcoI restriction site, and was transiently transformed into mesophyll protoplasts from four-week-old $A$. thaliana leaves. After transformation in darkness at $22^{\circ} \mathrm{C}$ for $20 \mathrm{~h}$, YFP signals were evaluated using a Zeiss LSM 510 Meta confocal microscope (Carl Zeiss, Jena, Germany) with an excitation wavelength of $514 \mathrm{~nm}$.

\subsection{Statistical Analysis}

IBM SPSS statistics software version 22.0 for Windows (IBM Corp., Armonk, NY, USA) was used to carry out one-way analysis of variance (ANOVA) among different samples using three replications. Duncan's multiple range test (DMRT) was used to determine significant differences $(p<0.05)$. 


\section{Conclusions}

In this study, we reported on the identification of 34 DoTPS genes in D. officinale. Their conserved motifs, exon-intron distribution, and phylogenetic analysis was assessed. Differential expression patterns of DoTPS genes exposed to ten different organs and three flowering stages, highlights their involvement in regulating the biosynthesis of floral monoterpenes, as well as the responses of plants to exogenous MeJA treatment, cold, and osmotic stress. One monoterpene synthase (DoTPS10), which was targeted to chloroplasts, could specifically convert GPP into linalool in vitro. Our findings show that transcript accumulation of multiple TPS genes is mainly responsible for the formation of floral terpenes, and provides a foundation for further studies on orchid floral scent research through the regulation of DoTPS genes.

Supplementary Materials: Supplementary materials can be found at http://www.mdpi.com/1422-0067/21/15/ 5419/s1.

Author Contributions: Conceptualization, Z.Y. and J.A.T.d.S.; methodology, Z.Y.; validation, Z.Y., C.Z., G.Z.; formal analysis, G.Z.; investigation, Z.Y.; resources, J.D.; data curation, Z.Y. and C.Z.; writing-original draft preparation, Z.Y., C.Z. and J.A.T.d.S.; writing-review and editing, J.A.T.d.S. and Z.Y.; visualization, Z.Y. and J.A.T.d.S.; supervision, J.D. and J.A.T.d.S.; project administration, J.D.; funding acquisition, J.D. All authors have read and agreed to the published version of the manuscript.

Funding: This research was funded by the National Key Research and Development Program of China, grant number 2018YFD1000400 and National Natural Science Foundation of China, grant number 31871547.

Acknowledgments: The authors are grateful to Yongxia Jia and Yangyang Xiao for assistance with GC-MS analysis of terpene compounds. We thank all the colleagues in our laboratory for help and advice. We would like to thank both the editor and reviewers for evaluating and providing constructive comments on this manuscript.

Conflicts of Interest: The authors declare no conflicts of interest.

\section{Abbreviations}

$\begin{array}{ll}\text { AI } & \text { Aliphatic index } \\ \text { bp } & \text { Base pair } \\ \text { CA } & \text { Cold acclimation } \\ \text { DMAPP } & \text { Dimethylallyl diphosphate } \\ \text { FPP } & \text { Farnesyl diphosphate } \\ \text { FPKM } & \text { Fragments per kilobase of transcript per million fragments mapped } \\ \text { GC-MS } & \text { Gas chromatography-mass spectrometry } \\ \text { GGPP } & \text { Geranylgeranyl diphosphate } \\ \text { GPP } & \text { Geranyl diphosphate } \\ \text { GRAVY } & \text { Grand average of hydrophobicity } \\ \text { II } & \text { Instability index } \\ \text { IPP } & \text { Isopentenyl diphosphate } \\ \text { MeJA } & \text { Methyl jasmonate } \\ \text { MEP } & \text { Methylerythritol phosphate } \\ \text { MS } & \text { Murashige and Skoog medium } \\ \text { Mw } & \text { Molecular weight } \\ \text { MVA } & \text { Mevalonic acid } \\ \text { NCBI } & \text { National Center for Biotechnology Information } \\ \text { NJ } & \text { Neighbor-joining } \\ \text { ORF } & \text { Open reading frame } \\ \text { pI } & \text { Isoelectric point } \\ \text { RT-qPCR } & \text { Real-time reverse transcription quantitative polymerase chain reaction } \\ \text { SDS-PAGE } & \text { Sodium dodecyl sulfate polyacrylamide gel electrophoresis } \\ \text { TPS } & \text { Terpene synthase } \\ \text { YFP } & \text { Yellow fluorescent protein }\end{array}$




\section{References}

1. Chen, F.; Tholl, D.; Bohlmann, J.; Pichersky, E. The family of terpene synthases in plants: A mid-size family of genes for specialized metabolism that is highly diversified throughout the kingdom. Plant J. 2011, 66, 212-229. [CrossRef] [PubMed]

2. Byers, K.J.; Bradshaw, H.D., Jr.; Riffell, J.A. Three floral volatiles contribute to differential pollinator attraction in monkeyflowers (Mimulus). J. Exp. Biol. 2014, 217, 614-623. [CrossRef] [PubMed]

3. Dudareva, N.; Klempien, A.; Muhlemann, J.K.; Kaplan, I. Biosynthesis, function and metabolic engineering of plant volatile organic compounds. New Phytol. 2013, 198, 16-32. [CrossRef] [PubMed]

4. Huang, W.; Gfeller, V.; Erb, M. Root volatiles in plant-plant interactions II: Root volatiles alter root chemistry and plant-herbivore interactions of neighbouring plants. Plant Cell Environ. 2019, 42, 1964-1973. [CrossRef]

5. Campbell, D.R.; Sosenski, P.; Raguso, R.A. Phenotypic plasticity of floral volatiles in response to increasing drought stress. Ann. Bot. 2019, 123, 601-610. [CrossRef]

6. Hsiao, Y.Y.; Pan, Z.J.; Hsu, C.C.; Yang, Y.P.; Hsu, Y.C.; Chuang, Y.C.; Shih, H.H.; Chen, W.H.; Tsai, W.C.; Chen, H.H. Research on orchid biology and biotechnology. Plant Cell Physiol. 2011, 52, 1467-1486. [CrossRef]

7. Ramya, M.; Jang, S.; An, H.R.; Lee, S.Y.; Park, P.M.; Park, P.H. Volatile organic compounds from orchids: From synthesis and function to gene regulation. Int. J. Mol. Sci. 2020, 21, 1160. [CrossRef]

8. Vranová, E.; Coman, D.; Gruissem, W. Network analysis of the MVA and MEP pathways for isoprenoid synthesis. Annu. Rev. Plant Biol. 2013, 64, 665-700. [CrossRef]

9. Tholl, D. Biosynthesis and biological functions of terpenoids in plants. Adv. Biochem. Eng. Biotechnol. 2015, 148, 63-106.

10. Bohlmann, J.; Meyer-Gauen, G.; Croteau, R. Plant terpenoid synthases: Molecular biology and phylogenetic analysis. Proc. Natl. Acad. Sci. USA 1998, 95, 4126-4133. [CrossRef]

11. Jiang, S.Y.; Jin, J.; Sarojam, R.; Ramachandran, S.A. Comprehensive survey on the terpene synthase gene family provides new insight into its evolutionary patterns. Genome Biol. Evol. 2019, 11, 2078-2098. [CrossRef] [PubMed]

12. Aubourg, S.; Lecharny, A.; Bohlmann, J. Genomic analysis of the terpenoid synthase (AtTPS) gene family of Arabidopsis thaliana. Mol. Genet. Genom. 2002, 267, 730-745. [CrossRef] [PubMed]

13. Zhou, H.C.; Shamala, L.F.; Yi, X.K.; Yan, Z.; Wei, S. Analysis of terpene synthase family genes in Camellia sinensis with an emphasis on abiotic stress conditions. Sci. Rep. 2020, 10, 933. [CrossRef] [PubMed]

14. Keilwagen, J.; Lehnert, H.; Berner, T.; Budahn, H.; Nothnagel, T.; Ulrich, D.; Dunemann, F. The terpene synthase gene family of carrot (Daucus carota L.): Identification of QTLs and candidate genes associated with terpenoid volatile compounds. Front. Plant Sci. 2017, 8, 1930. [CrossRef]

15. Külheim, C.; Padovan, A.; Hefer, C.; Krause, S.T.; Köllner, T.G.; Myburg, A.A.; Degenhardt, J.; Foley, W.J. The Eucalyptus terpene synthase gene family. BMC Genom. 2015, 16, 450. [CrossRef]

16. Nieuwenhuizen, N.J.; Green, S.A.; Chen, X.; Bailleul, E.J.D.; Matich, A.J.; Wang, M.Y.; Atkinson, R.G. Functional genomics reveals that a compact terpene synthase gene family can account for terpene volatile production in apple. Plant Physiol. 2013, 161, 787-804. [CrossRef]

17. Falara, V.; Akhtar, T.A.; Nguyen, T.T.H.; Spyropoulou, E.A.; Bleeker, P.M.; Schauvinhold, I.; Matsuba, Y.; Bonini, M.E.; Schilmiller, A.L.; Last, R.L.; et al. The tomato terpene synthase gene family. Plant Physiol. 2011, 157, 770-789. [CrossRef]

18. Li, G.; Köllner, T.G.; Yin, Y.; Jiang, Y.; Chen, H.; Xu, Y.; Gershenzon, J.; Pichersky, E.; Chen, F. Nonseed plant Selaginella moellendorffii has both seed plant and microbial types of terpene synthases. Proc. Natl. Acad. Sci. USA 2012, 109, 14711-14715. [CrossRef]

19. Martin, D.M.; Aubourg, S.; Schouwey, M.B.; Daviet, L.; Schalk, M.; Toub, O.; Lund, S.T.; Bohlmann, J. Functional annotation, genome organization and phylogeny of the grapevine (Vitis vinifera) terpene synthase gene family based on genome assembly, FLcDNA cloning, and enzyme assays. BMC Plant Biol. 2010, 10, 226. [CrossRef]

20. Chuang, Y.C.; Hung, Y.C.; Tsai, W.C.; Chen, W.H.; Chen, H.H. PbbHLH4 regulates floral monoterpene biosynthesis in Phalaenopsis orchids. J. Exp. Bot. 2018, 69, 4363-4377. [CrossRef]

21. Gao, F.; Liu, B.; Li, M.; Gao, X.; Fang, Q.; Liu, C.; Ding, H.; Wang, L.; Gao, X. Identification and characterization of terpene synthase genes accounting for volatile terpene emissions in flowers of Freesia $\times$ hybrida. J. Exp. Bot. 2018, 69, 4249-4265. [CrossRef] [PubMed] 
22. Yan, L.; Wang, X.; Liu, H.; Tian, Y.; Lian, J.; Yang, R.; Hao, S.; Wang, X.; Yang, S.; Li, Q.; et al. The genome of Dendrobium officinale illuminates the biology of the important traditional Chinese orchid herb. Mol. Plant 2015, 8, 922-934. [CrossRef] [PubMed]

23. Zhang, G.; Xu, Q.; Bian, C.; Tsai, W.C.; Yeh, C.M.; Liu, K.; Yoshida, K.; Zhang, L.; Chang, S.; Chen, F.; et al. The Dendrobium catenatum Lindl genome sequence provides insights into polysaccharide synthase, floral development and adaptive evolution. Sci. Rep. 2016, 6, 1-10. [CrossRef] [PubMed]

24. Tsai, W.C.; Dievart, A.; Hsu, C.C.; Hsiao, Y.Y.; Chiou, S.Y.; Huang, H.; Chen, H.H. Post genomics era for orchid research. Bot. Stud. 2017, 58, 61. [CrossRef] [PubMed]

25. Zhang, G.Q.; Liu, K.W.; Li, Z.; Lohaus, R.; Hsiao, Y.Y.; Niu, S.C.; Wang, J.Y.; Lin, Y.C.; Xu, Q.; Chen, L.J.; et al. The Apostasia genome and the evolution of orchids. Nature 2017, 549, 379-383. [CrossRef]

26. Kaundal, R.; Saini, R.; Zhao, P.X. Combining machine learning and homology-based approaches to accurately predict subcellular localization in Arabidopsis. Plant Physiol. 2010, 154, 36-54. [CrossRef]

27. Chou, K.C.; Shen, H.B. Plant-mPLoc: A top-down strategy to augment the power for predicting plant protein subcellular localization. PLOS ONE. 2010, 5, 11335. [CrossRef]

28. Cheng, X.; Xiao, X.; Chou, K.C. PLoc-mPlant: Predict subcellular localization of multi-location plant proteins by incorporating the optimal GO information into general PseAAC. Mol. Biosyst. 2017, 13, 1722-1727. [CrossRef]

29. Wu, Z.; Jiang, W.; Chen, S.; Mantri, N.; Tao, Z.; Jiang, C. Insights from the cold transcriptome and metabolome of Dendrobium officinale: Global reprogramming of metabolic and gene regulation networks during cold acclimation. Front. Plant Sci. 2016, 7, 1653. [CrossRef]

30. Teixeira da Silva, J.A.; $\mathrm{Ng}$, T.B. The medicinal and pharmaceutical importance of Dendrobium species. Appl. Microbiol. Biotechnol. 2017, 101, 2227-2239. [CrossRef]

31. Yu, Z.; Yang, Z.; Teixeira da Silva, J.A.; Luo, J.; Duan, J. Influence of low temperature on physiology and bioactivity of postharvest Dendrobium officinale stems. Postharvest Biol. Tech. 2019, 148, 97-106. [CrossRef]

32. Wan, X.; Zou, L.; Zheng, B.; Tian, Y.; Wang, Y. Transcriptomic profiling for prolonged drought in Dendrobium catenatum. Sci. Data 2018, 5, 180233. [CrossRef] [PubMed]

33. Kaul, S.; Koo, H.L.; Jenkins, J.; Rizzo, M.; Rooney, T.; Tallon, L.J.; Feldblyum, T.; Nierman, W.; Benito, M.I.; Town, C.D.; et al. The Arabidopsis Genome Initiative. Analysis of the genome sequence of the flowering plant Arabidopsis thaliana. Nature 2000, 408, 796-815.

34. Banks, J.A.; Nishiyama, T.; Hasebe, M.; Bowman, J.L.; Gribskov, M.; de Pamphilis, C.; Albert, V.A.; Aono, N.; Aoyama, T.; Ambrose, B.A.; et al. The Selaginella genome identifies genetic changes associated with the evolution of vascular plants. Science 2011, 332, 960-963. [CrossRef]

35. Huang, X.; Kurata, N.; Wei, X.; Wang, Z.X.; Wang, A.; Zhao, Q.; Zhao, Y.; Liu, K.; Lu, H.; Li, W.; et al. A map of rice genome variation reveals the origin of cultivated rice. Nature 2012, 490, 497-501. [CrossRef]

36. Jaillon, O.; Aury, J.M.; Noel, B.; Policriti, A.; Clepet, C.; Casagrande, A.; Choisne, N.; Aubourg, S.; Vitulo, N.; Jubin, C.; et al. French-Italian Public Consortium for Grapevine Genome Characterization. The grapevine genome sequence suggests ancestral hexaploidization in major angiosperm phyla. Nature 2007, 449, 463-467.

37. Hong, G.J.; Xue, X.Y.; Mao, Y.B.; Wang, L.J.; Chen, X.Y. Arabidopsis MYC2 interacts with DELLA proteins in regulating sesquiterpene synthase gene expression. Plant Cell 2012, 24, 2635-2648. [CrossRef]

38. Murashige, T.; Skoog, F. A revised medium for rapid growth and bioassays with tobacco tissue cultures. Physiol. Plantarum 1962, 15, 473-497. [CrossRef]

39. Saitou, N.; Nei, M. The neighbor-joining method: A new method for reconstructing phylogenetic trees. Mol. Biol. Evol. 1987, 4, 406-425.

40. Kumar, S.; Stecher, G.; Tamura, K. MEGA7: Molecular evolutionary genetics analysis version 7.0 for bigger datasets. Mol. Biol. Evol. 2016, 33, 1870-1874. [CrossRef]

41. Yu, Z.; He, C.; Teixeira da Silva, J.A.; Luo, J.; Yang, Z.; Duan, J. The GDP-mannose transporter gene (DoGMT) from Dendrobium officinale is critical for mannan biosynthesis in plant growth and development. Plant Sci. 2018, 277, 43-54. [CrossRef]

42. An, H.; Zhu, Q.; Pei, W.; Fan, J.; Liang, Y.; Cui, Y.; Lv, N.; Wang, W. Whole-transcriptome selection and evaluation of internal reference genes for expression analysis in protocorm development of Dendrobium officinale Kimura et Migo. PLoS ONE 2016, 11, 163478. [CrossRef] [PubMed]

43. Livak, K.J.; Schmittgen, T.D. Analysis of relative gene expression data using real-time quantitative PCR and the $2^{-\Delta \Delta C T}$ method. Methods 2001, 25, 402-408. [CrossRef] [PubMed] 
44. Yu, Z.; Zhang, G.; Teixeira da Silva, J.A.; Yang, Z.; Duan, J. The $\beta$-1,3-galactosetransferase gene DoGALT2 is essential for stigmatic mucilage production in Dendrobium officinale. Plant Sci. 2019, 287, 110179. [CrossRef] [PubMed]

45. Yu, Z.; Liao, Y.; Zeng, L.; Dong, F.; Watanabe, N.; Yang, Z. Transformation of catechins into theaflavins by upregulation of CSPPO3 in preharvest tea (Camellia sinensis) leaves exposed to shading treatment. Food Res. Int. 2020, 129, 108842. [CrossRef] [PubMed]

(C) 2020 by the authors. Licensee MDPI, Basel, Switzerland. This article is an open access article distributed under the terms and conditions of the Creative Commons Attribution (CC BY) license (http://creativecommons.org/licenses/by/4.0/). 